\title{
Die Politik in der politischen Gesellschaft. Eine ideengeschichtliche Spurensuche. In memoriam Michael Th. Greven (07.03.1947- 07.07.2012).
}

\author{
Friedbert W. Rüb
}

Politics in the Political Society. Investigating Traces in the History of Political Ideas. In memoriam Michael Th. Greven (07.03.1947-07.07.2012).

Summary: The "political society" (Greven) presupposes politics as the main authority capable of shaping society and giving it political order. Politics alone are able to bind together (post)modern societies and in principle intervene in all societal spheres and can virtually politicize everything. In spite of its central position, the term "politics" seems to be peculiarly empty. This contribution tries to investigate the traces of the term politics. It starts with that point in time when the term was rewritten and newly conceptualized at the turning point to modernity and reconstructs the new idea of politics using the work of four authors, namely Albert E. F. Schäffle, Karl Mannheim, Max Weber, and Niklas Luhmann. All refer to each other explicitly or implicitly and begin to constitute a web of a political discourse in which a new idea of politics originates. It displays a new outline of politics as political action that is appropriate to the high significance of politics in the "political society".

Keywords: Politicization, politicking, political society, politics

Schlagwörter: Politik, Politisierung, politische Gesellschaft

\section{Einleitung'}

Die „politische Gesellschaft”, die Michael Th. Greven (1999) als politische Theorie, ja als Gegentheorie zu verschiedenen sozialwissenschaftlichen Gesellschaftstheorien und anderen Zeitdiagnostiken beschrieben hat, sieht in der Politik die zentrale Ordnungs- und Gestaltungsinstanz. Politische Entscheidungen halten moderne Gesellschaften nicht nur organisatorisch und normativ zusammen, sondern stecken zugleich auch den Möglichkeitshorizont ab, in dem sie sich selbst denken und über sich selbst entscheiden können. Dabei ist, wie bei allen Entscheidungen, Dezisionismus unhintergehbar, er ist der Schatten, der sie immer begleitet. Es sind also weder vorpolitische, kulturelle oder soziologische Sachverhalte noch funktional ausdifferenzierte, autopoietisch operierende Systeme mit loser Kopplung, noch kommunikativ ermittelte Vernunft, die moderne Gesellschaften integrieren. Vielmehr sind es kontingente politische Entscheidungen, die in ihrer

1 Für wichtige Hinweise danke ich meinen Mitarbeiter/innen Holger Straßheim, Tom Ulbricht, Yasmin Hilpert und David Meiering, sowie Jan-Christoph Rogge ebenso wie zwei anonymen Gutachtern bzw. Gutachterinnen der PVS. 
potenziellen Reichweite unbegrenzt sind und deshalb die demokratische Gestaltbarkeit von Gesellschaften ebenso einschließen wie die extrem destruktive Selbstzerstörung, die in den beiden großen Totalitarismen des 20. Jahrhunderts zum Ausdruck kam.

Politik ist in all ihrer Ambivalenz das Schicksal der Moderne. Aber der Politikbegriff selbst bleibt in der politischen Gesellschaft trotz seiner Zentralstellung eigentümlich unbestimmt. Wenn Politik das Schicksal moderner Gesellschaften ist - im Guten wie im Bösen - so ist diese ,Leerstelle' einigermaßen erstaunlich. Um nicht missverstanden zu werden: An vielen Stellen der politischen Gesellschaft und auch an anderen Stellen in dem weit verstreuten Werk von Michael Th. Greven finden sich überraschende und erhellende Beobachtungen und Bemerkungen zum Politikbegriff, aber eine grundlegende Ausarbeitung - sei sie ideengeschichtlich, systematisch oder typologisch - hat er nicht geleistet. ${ }^{2}$

In den folgenden Überlegungen will ich genau dies v. a. ideengeschichtlich versuchen und riskiere einen spezifischen Zugriff auf das Phänomen der Politik: Ich unternehme eine Spurensuche und verfolge ausschließlich die Spuren des Politikbegriffs, der sich in der gegenseitigen Bezugnahme von Autoren um die Wende vom 19. zum 20. Jahrhundert, also von der Prämoderne zur Moderne (dazu von Beyme 1991, S. 29-43), beobachten lässt. Ich springe dann in die sechziger Jahre des 20. Jahrhunderts, also etwa in den Übergang von der Moderne zur Postmoderne (von Beyme 1991), weil es hier einen Autor gab, der diese Diskussionen rekonstruiert, in seinem Werk über den Politikbegriff darauf Bezug nimmt, sich mit diesen Autoren kritisch auseinandersetzt und zugleich eine Neuorientierung vornimmt, die die alten Spuren aber nicht gänzlich abstreift. Rund 25 Jahre später erscheint dann erneut eine Schrift desselben Autors, die ebenfalls die Politik zum Gegenstand hat und in der in aller Schärfe ein postmoderner Politikbegriff formuliert wird, der in seiner Radikalität das bisherige Verständnis erneut umschreibt.

Die Begründung für eine Spurensuche und gegen eine systematische Rekonstruktion des Politikbegriffs liegt darin, dass sich in diesen historischen Phasen Umund Neuschreibungen des Politikbegriffs finden lassen müssten, die Greven seinem Politikbegriff implizit zugrunde legt. Denn wenn die politische Gesellschaft geschichtlich im Übergang vom 19. zum 20. Jahrhundert zum Durchbruch kam, dann müssten sich in den zeitgenössischen Diskussionen Formulierungen finden lassen, die das Umschreiben des Politikbegriffs vom Substanz- zum Handlungsbegriff widerspiegeln. Die Konzeptionen von Politik als Kontingenzproduktion, als dezisionistisch angelegtes Entscheiden, als Fundamentalpolitisierung und schließlich als Schicksal moderner Gesellschaften müssten in diesen Texten ihre Spuren hinterlassen.

Diese Autoren sind - und das mag auf den ersten Blick erstaunen - Albert E. F. Schäffle, Karl Mannheim, Max Weber und Niklas Luhmann. Die ersten drei markieren den Moment, in dem der Politikbegriff neu konturiert wird, signalisieren einen Zeit- oder Wendepunkt, ab dem ein neuer, bisher nicht dagewesener Aspekt im Politikbegriff auftaucht und dann die weitere Diskussion bestimmt. Im Gegen-

2 Ich weiß aus vielen Gesprächen, dass er dies für sein Werk und für die Politikwissenschaft für dringend nötig hielt, aber aus den verschiedensten Gründen nicht leisten konnte. 
satz zu dem finnischen Politiktheoretiker Kari Palonen gehe ich hier nicht vom „Weber'schen Moment“ aus (Palonen 1998), sondern vom Schäffle'schen Moment. Schäffle hat bereits 1897 in dem Artikel „Über den wissenschaftlichen Begriff der Politik“ (Schäffle 1897) den Anfang gesetzt.

Karl Mannheim ist ihm in seinen Überlegungen über „Kann es Wissenschaft von der Politik geben“ (Mannheim [1929] 1952) gefolgt und hat gleichzeitig neue zentrale Aspekte für den Politikbegriff hinzugefügt und Politik als „irrationales Spiel“ (Mannheim [1929] 1952, S. 99) bezeichnet. Max Weber hat in seinem frühen Vortrag im Jahr 1919 an der Münchener Hochschule über „Politik als Beruf“ (Weber [1919] 1992) keinen einzigen Hinweis auf Albert E. F. Schäffle gegeben, verwendet aber wortgleiche Formulierungen, die m. E. nur auf ihn zurückgehen können. Er müsste diese Schrift also bei der Entwicklung seiner Vorstellungen gekannt haben. Diese drei Autoren markieren je unterschiedlich den Übergang von der Vormoderne zur Moderne, während Niklas Luhmann für den Übergang von der Moderne zur Postmoderne steht. Bei ihm beziehe ich mich v. a. auf seine Mitte der sechziger Jahre gehaltene Vorlesung über „Politische Soziologie“ (Luhmann 2010) und nicht auf die „Politik der Gesellschaft“ (Luhmann 2000). Die Gründe hierfür sind klar: Ich brauche für eine Spurensuche ausdrückliche Bezüge von Niklas Luhmann auf Albert E. F. Schäffle, Karl Mannheim und Max Weber, die bei Niklas Luhmann nur in dieser und - wenn ich das recht sehe - in keiner anderen Schrift zu finden sind. Hier entwickelt er in expliziter und impliziter Auseinandersetzung mit ihnen seine (frühe) Systemtheorie der Politik. Durch die Verweise zwischen ihnen verbinden sich diese Diskussionslinien zu einem „Gewebe politischer Diskurse“ (Llanque 2008), die sich auf eine (Neu)Konturierung des Politikbegriffs konzentrieren, die extrem folgenreich ist, aber im Mainstream der Politikwissenschaft erstaunlich unberücksichtigt blieb. Und erst in der „Politik der Gesellschaft", die Anfang der neunziger Jahre geschrieben wurde, ${ }^{3}$ vollzieht Niklas Luhmann die radikale Wende zu einem postmodernen Politikbegriff.

Ich gehe wie folgt vor: In einem ersten Schritt rekonstruiere ich in kritischer Absicht die Grundprämissen der politischen Gesellschaft ebenso wie ihre vier historischen Voraussetzungen. Die Grundidee der Fundamentalpolitisierung geht und dies mag auf den ersten Blick erstaunen - auf den Bonner Zeithistoriker und Politologen Karl D. Bracher zurück, bei dem Greven promoviert hat (2.). Danach trage ich all die Bemerkungen zusammen, die für den Politikbegriff der politischen Gesellschaft zentral sind, und greife hierbei auch auf andere Schriften zurück, in denen sich Michael Th. Greven dazu geäußert hat (3.). Anschließend rekonstruiere ich - selektiv und zum Teil als Spurensuche in den Fußnoten der jeweiligen Autoren - das Politikverständnis von Albert E. F. Schäffle, Karl Mannheim und Max Weber, und schließlich kommt Niklas Luhmann durch seine „Politische Soziologie“ zu Wort (4.). Eine Zusammenfassung beendet meine Überlegungen, in der ich abschließend und sporadisch den postmodernen Politikbegriff von Niklas Luhmann andeute (5.).

3 Vgl. dazu die editorische Notiz des Herausgebers A. Kieserling in Luhmann 2000, S. 435-437. 


\section{Die „politische Gesellschaft" als Zeitdiagnose und Gesellschaftstheorie}

Alles hat seine Geschichte - das gilt auch für die Idee der politischen Gesellschaft. Wir stehen alle auf den Schultern von Riesen (Merton 1980); deshalb kann auch ein Zwerg weiter sehen als der Riese selbst, sofern er auf dessen Schultern steht. Eine neue wissenschaftliche Erkenntnis entsteht nicht aus dem Nichts, sondern immer in einem historischen Kontext, der durch Riesen konstituiert wird und von deren Wissen man sich entweder in kritischer Auseinandersetzung absetzt oder deren Kenntnisse man für seine eigene Theorieproduktion verwendet.

Die ersten Grundideen für die „Politische Gesellschaft“ hat Greven in seiner 1974 erschienenen und bei Karl D. Bracher geschriebenen Doktorarbeit entwickelt. In ihr setzt er sich in kritischer Absicht mit verschiedenen systemtheoretischen und kybernetischen Gesellschaftstheorien auseinander (Greven 1974a, konzentriert 1974b). Der Systemtheorie mit ihrer zentralen Kategorie des Überlebens (eines Status Quo von Systemen durch Adaption) setzt er ein Denken entgegen, das sich an der Kategorie des real Möglichen orientiert und von hier aus die gesellschaftsverändernden Potentiale einer demokratischen Gesellschaft auslotet. Demokratie wird hier als „explizite Wertprämisse“ eingeführt, die - von ihm radikal-demokratisch definiert - den Möglichkeitshorizont einer Gesellschaft hinsichtlich ihrer materiellen Ressourcen und ihrer Werte und Prinzipien ausleuchtet und das in ihr denkbar zukünftige Mögliche reflektiert. Dieses denkbar Mögliche umfasst zwei Dimensionen. Zunächst die Idee von „basic human needs“(Greven 1974a, S. 40-46), die eine demokratische Gesellschaft befriedigen muss. Und zweitens die eines freien und selbstbestimmten Handelns jenseits gesellschaftlich bedingter Entfremdung, um die Potentiale einer „kontingenten Zukunft“ durch politische Praxis zu realisieren (Greven 1974a, S. 49). Aktive und kritische Partizipation sind hier die entsprechenden Instrumente. Demokratie bedeutet dann die „weitmöglichste Freiheit von irrationalen Zwängen, von Herrschaft also, die immer Herrschaft von Menschen, niemals von Sachen allein ist, allenfalls von in eindeutigen Herrschaftsverhältnissen als unabänderliche ,Sachzwänge‘ interpretierten Verhältnissen, die veränderbar sind, sich als veränderbar erweisen, wenn sie einer kritischen Reflexion unterzogen werden, die ihre lediglich perspektivisch bedingte Unabänderlichkeit relativiert" (Greven 1974a, S. 37).

Unter einer solchen partizipativen und reflexiven Perspektive wird auch der Politikbegriff gedacht. Politik ist hier zunächst der Prozess der Politisierung, denn die (damalige) Gesellschaft war durch die zunehmende Politisierung von Bereichen gekennzeichnet, die bisher als unpolitisch galten und nun als Bewusstwerdung längst bestehender Herrschaftsverhältnisse thematisiert wurden. Politik, die „von oben“ wie „von unten“ in Gang gesetzt werden kann, hat hier v. a. die Aufgabe der Öffnung des Möglichkeitshorizonts. In der Politik moderner Gesellschaften gibt es grundsätzlich keinen der Politik entzogenen Bereich mehr, im Prinzip ist alles politisierbar und im Prinzip kann alles zum Gegenstand einer politischen Entscheidung gemacht werden. Während hier noch von politisierten Gesellschaften die Rede ist, wird daraus später die „politische Gesellschaft“, die diese Grundidee radikalisiert. Der zentrale Bezugspunkt - oder der Riese, auf dessen Schultern Michael Th. Greven steht - ist Karl D. Bracher. Er taucht als ,Vater 
dieser Idee zwar nur in einer Fußnote auf, aber in ihr findet sich das zentrale $\mathrm{Zi}$ tat, das ihn so stark beeinflusst und zur zentralen Prämisse der politischen Gesellschaft wird: „Es gibt in der modernen Demokratie wie auf anderer Seite in den totalitären Staaten keinen der Politik völlig entzogenen und entziehbaren Bereich" (Bracher 1964, S. 121). Es ist eher ein kommentierender Nebensatz in einem Kontext, der die Bedeutung der Interessengruppen im Nachkriegsdeutschland und deren Politisierungskraft hervorhebt. Aber wie in einem Brennglas ist die Botschaft formuliert: Keinen entzogenen Bereich - das heißt, alles ist bereits weitgehend politisiert; keinen entziehbaren Bereich - es gibt keine prinzipiellen Grenzen, an denen die Politik Halt macht und die ihre Politisierungsdynamiken beschränken. Und deshalb sind auch totalitäre Regime ein Subtypus der politischen Gesellschaft, in denen alles politisiert ist und Politik (samt ihrem Gewaltpotential) entgrenzt ist. ${ }^{4}$

Anfang der neunziger Jahre beginnen Michael Th. Grevens zentrale Arbeiten zur politischen Gesellschaft, die ihren Abschluss mit dem Buch „Die politische Gesellschaft" finden (1999). Bereits 1990 erschien in der inzwischen eingestellten Zeitschrift „Ethik und Sozialwissenschaft. Streitforum für Erwägungskultur“ (was für ein Untertitel!) sein Aufsatz „Die politische Gesellschaft als Gegenstand der Politikwissenschaft" (Greven 1990). Auf diesen Essay, dem ein Bewerbungsvortrag für eine Soziologieprofessur in Bielefeld zugrunde lag, haben dann etliche PolitikwissenschaftlerInnen mit zum Teil massiver Kritik und manch grobem Unverständnis reagiert. ${ }^{5}$ Aber hier werden in Nuancen bereits alle zentralen Prämissen des späteren Buches formuliert.

Politische Gesellschaften sind unter bestimmten historischen Bedingungen entstanden und vier interdependente Entwicklungen sind dafür zentral: (a) Zunächst Säkularisierung, in der jede politische Herrschaft nur noch aus sich selbst gerechtfertigt werden kann und aller religiösen oder naturrechtlichen Legitimationsquellen verlustig geht. Moderne Politik muss „,selbstragend““ sein (Greven 1999, S. 36), denn sie ruht auf keinem anderen Fundament mehr auf. Alle Standards und Normen der Politik, seien sie rechtlicher oder kultureller Art, müssen politisch kreiert werden - nur über die Selbst-Institutionalisierung von „Fesseln und Bremsen“ (Offe 1989) wird Politik nicht übermächtig. Dann ist (b) Interessenreduktion als Rationalisierung der Mechanismus, der Leidenschaften, Emotionen, Aufwallungen und Unbeherrschtheiten der Individuen (und deren Vertretungen) in Interessen verwandelt, die man verhandeln, aggregieren, teilen und in Kompromisse überführen kann (Greven 1999, S. 50-66). Leidenschaften, Ideologien oder Identitäten sind dagegen Kompromissen kaum zugänglich. Und diese Konvertabilität in Interessen erzeugt Kontingenz und Flexibilität der Politik (und der in der Politik engagierten Akteure), die bis zur Gesinnungslosigkeit reichen kann (Gre-

4 Zur Differenzierung von politischen Regimetypen lesbar und erhellend Merkel 2010.

5 Vgl. insbesondere die Repliken von Johannes Agnoli, Heinrich Bußhoff, Ulrich Druwe, Rolf Ebbinghaus, Jürgen Gebhardt, Gerhard Göhler, Axel Görlitz, Werner Patzelt und Bernhard Willms. Es ist interessant, diese Kritikpunkte heute erneut zu lesen, denn Vieles, was damals kritisiert wurde, ist heute in der Disziplin common sense und würde keine so massive Kritik mehr hervorrufen. Aber ebenso interessant wäre eine Analyse der zum Teil völlig überzogenen Kritik. 
ven 1999, S. 50). ${ }^{6}$ (c) Woblfahrtsstaatliche Inklusion bedeutet die Verstaatlichung der Gesellschaft in dem Sinne, dass es keine „politiklose“ Gesellschaft mehr gibt. Vielmehr ist sie von einem robusten Dauergerüst politischer Entscheidungen durchzogen. Kapitalistische Ökonomie und andere Teilsysteme der Gesellschaft folgen zwar ihrer je eigenen Logik, aber sie sind politisch konstituiert und konfirmiert und nicht durch rein evolutionäre Entwicklung entstanden. Die Lebenslage von ganzen „Versorgungsklassen“ des Wohlfahrtsstaates hängt von politischen Entscheidungen ebenso ab wie die Verteilungsmuster der sozialen Ungleichheit (Greven 1999, S. 62-63). Die Politik wirft ein immer engmaschigeres Netz über die Gesellschaft, wodurch die Gesellschaft und das individuelle Leben nun zweischalig geformt werden: Einmal durch die rein individuellen Tätigkeiten und Entscheidungen und andererseits durch wohlfahrtsstaatliche Institutionen, die ein breites Geflecht von Regelungen bereitstellen, innerhalb dessen sich das moderne Leben vollzieht. Und schließlich (d) als historisch letzter Schritt die Politisierung zentraler gesellschaftlicher Bereiche: „Von oben“ durch die bereits historisch entstandenen Verwaltungsbeamten und Politiker, die sich nun dem Konstitutionalismus und der horizontalen Gewaltenteilung unterwerfen müssen und zugleich an den demokratischen Willen des Volkes rückgebunden werden. „Von unten“ durch die Einräumung des allgemeinen und gleichen Wahlrechts, das erst spät erkämpft wurde und die Massen politisierte. Sie werden nun zu politischen Subjekten der Geschichte, sei es durch demokratische Mitbestimmung in den pluralistisch-konstitutionellen Gesellschaften, sei es durch die (erzwungene) Massenmobilisierung in den totalitären Regimen (Greven 1999, S. 70).

Politische Gesellschaften sind somit erst in einem bestimmten historischen Moment entstanden und können deshalb ihren Charakter wieder verlieren, weil sie ein kontingentes Phänomen sind. Doch was sind die definitorischen Merkmale, die sie als genuin „politisch“ auszeichnen? Als historisch beobachtbare und erst in der Moderne sich voll entwickelnde Gesellschaftsformation ist sie durch fünf zentrale Merkmale gekennzeichnet:

(i) Zunächst durch Fundamentalpolitisierung; denn in ihr ist

„Politik virtuell total geworden. Nichts ist in ihr prinzipiell unpolitisch geblieben, keine eigenständigen Bereiche, Sphären oder Systeme existieren, deren Politisierung ausgeschlossen wäre, wenn sie nicht bereits politisch geworden sind. [...] Politisch-

Sein [ist] zum entscheidenden Bestimmungsmerkmal, zum Wesen der heutigen Gesellschaften geworden, neben dem andere Qualitäten zurücktreten“ (Greven 1990, S. 223-224).

Die politische Gesellschaft ist ein spezifischer Typus der Vergesellschaftung, der funktional und historisch einzigartig ist und ihre Fundamentalpolitisierung ist nicht auf die tradierten Wege der Wahl angewiesen. Obwohl das allgemeine Wahlrecht ein zentraler Mechanismus ist, kann sie auch durch neue soziale Bewegungen, Proteste, Demonstrationen u. Ä. erfolgen, wobei die Qualität dieser Politisierungspraktiken von der Qualität der politischen Meinungs- und Willensbildung

6 Auffallend ist, dass der „Klassiker“ zu dieser Thematik von ihm nicht erwähnt wird: Albert O. Hirschmans „Leidenschaften und Interessen“ (Hirschman 1987). 
abhängt. Aber auch totalitäre Regime können Fundamentalpolitisierungen durch inszenierte Massenbewegungen ohne demokratische Grundrechte in Gang setzen, wie die Geschichte des 20. Jahrhunderts überdeutlich gezeigt hat (Greven 1990, S. 226-227, 1999, S. 54-60).

(ii) Damit untrennbar verbunden ist die Prämisse, dass politische Gesellschaften, v. a. der demokratisch-pluralistische Typus, nicht mehr auf einem wie auch immer gearteten Grundkonsens aufsitzen, sondern dass alle zentralen Werte und auch die Verteilung der gesellschaftlichen Ressourcen politisch entschieden werden müssen. Alle wichtigen Normen und alle Ungleichheiten erhalten ihre (relative) gesamtgesellschaftliche Verbindlichkeit allein dadurch, dass sie politisch entschieden sind und genau das macht ihren politischen Charakter aus. Deshalb müssen sich soziale Ordnungsmuster rechtfertigen und sie müssen rechtfertigbar sein, weil sie sonst nicht anerkannt werden. Grundsätzlich ist keine andere Möglichkeit der gesellschaftlichen Ordnungsbildung sichtbar, allein politische Entscheidungen vermögen dies, weil sie entweder legitim sind oder weil sie sich mit Macht oder legitimer physischer Gewaltsamkeit durchsetzen. Kulturen oder soziale Normen von Gruppen können diese Ordnungsbildung nicht leisten. Als Folge sind

(iii) alle Entscheidungen kontingent, denn jede Entscheidung hätte auch anders ausfallen können. Es gibt keine allgemein anerkannten, universalistischen Gründe für diese oder gegen andere mögliche Entscheidungen, sondern sie sind das Ergebnis spezifischer, sich wandelbarer Macht-, Verhandlungs- oder Diskurskonstellationen und fallen deshalb so und nicht anders aus. Zudem können sie - trotz Pfadabhängigkeiten - jederzeit teilweise oder ganz revidiert werden. Angesichts nicht übereinstimmender und konfligierender Wertorientierungen und Präferenzen von Einzelnen und Gruppen können verbindliche Entscheidungen diese Kontingenz nur temporär überwinden, weil sie allein aufgrund ihrer Legitimität oder Legalität, aber nicht wegen ihrer Richtigkeit oder Wahrheit gelten. Richtigkeit bzw. Vernunft und Geltung fallen unwiderruflich auseinander (Greven 1999, S. 66-67). Und schließlich ist damit - das ist der am massivsten angegriffene Punkt - ein

(iv) unhintergehbarer Dezisionismus verbunden, der sich aus der Komplexität und der Herausgehobenheit des Entscheidens ergibt. Denn „weder im Bewusstsein der relevanten Akteure noch von der Sache her [gab] es jemals so viel zu entscheiden wie heute" (Greven 1999, S. 61). Zudem erfolgt Entscheiden unter hoher Komplexität, die rationale Entscheidungen faktisch verunmöglicht und sowohl die Entscheider wie auch die mitentscheidenden Bürger vor unlösbare kognitive Probleme stellt. Willkürlichkeit ist unvermeidlich, weil man den Graben der Unsicherheit und des Nichtwissens bei jeder Entscheidung überspringen und sich festlegen muss. Zudem sind die Folgen politischer Entscheidungen nicht im Voraus zu bilanzieren, weil die Eigendynamiken und die Multikausalität aller sozialen und gesellschaftlichen Prozesse weder durch Alltags- noch durch wissenschaftliches Wissen aufgehoben werden können. Und schließlich ist das Widerstreben anderer politischer und nicht-politischer Gruppierungen dafür verantwortlich, dass Entscheidungen keine Sach-, sondern eben politische Entscheidungen sind, weil sie sich erst im Kampf herauskristallisieren und so ihre endgültige Form gewinnen (Greven 1992). Politisches Entscheiden ist zwar darauf nicht zu reduzie- 
ren, aber man kann es heute ohne (demokratischen) Dezisionismus nicht mehr denken. ${ }^{7}$ Daraus folgt

(v) ein wesentlich prozedurales Verständnis von Politik, das sich nicht auf die „offizielle“ Politik des politischen Systems reduzieren lässt, sondern als „polyzentrische politische Entscheidungsstruktur" 8 gedacht wird. Politik wird nicht von einer Regierung gemacht, sondern von einer „Vielzahl von Quasi-Regierungen, die in einem interdependenten Zusammenhang stehen " (Greven 1999, S. 92). Damit untrennbar verbunden ist die Vorstellung, dass die funktionale Differenzierung moderner Gesellschaften zwar empirische Realität ist, die Autopoiesis ihrer Teilsysteme dagegen eine theoretische Fiktion. Denn Politik kann „vermittels angedrohter oder ausgeübter Gewalt oder kommunikativem Zusammenwirken praktisch in alle gesellschaftlichen Bereiche intervenieren" (Greven 1999, S. 108). ${ }^{9}$ Interessant wird dann die Frage, warum und aufgrund welcher Selektions- oder Selbstbindungsmechanismen Politik nicht in alle gesellschaftlichen Bereiche eingreift, obwohl sie es könnte. Politik setzt sich in liberal-demokratischen Staaten durch Verfassungen und individuelle und politische Freiheitsrechte selbst Grenzen, die sie aber nur selbst errichten und deshalb auch jederzeit wieder zerstören kann. Politik - so schlussfolgert er - ist „jene Form der gesellschaftlichen Selbstregulierung, die sich als Folge bewusst getroffener und in ihrer Reichweite definierter verbindlicher Entscheidungen ergibt" (Greven 1999, S. 69). Die Dichotomie von „politischer Steuerung“ und "gesellschaftlicher Selbstregulierung“ (Mayntz u. Scharpf 1995) wird von ihm also nicht anerkannt, sondern durch einen Politikbegriff unterlaufen, der sich aus dem Fundamentalpolitisierungsparadigma ergibt.

\section{Grevens Politik in der politischen Gesellschaft}

Trotz der Sonderstellung der Politik sind der Politikbegriff und der des politischen Handelns bei Michael Th. Greven erstaunlich unterbelichtet. Dennoch: Seine Kritik richtet sich zunächst sowohl gegen funktionalistisch als auch gegen finalistisch

7 Zum Dezisionismus als eigenständigem Typus des politischen Handelns vgl. auch Bolsinger 1998.

8 Es ist interessant, dass Michael Th. Greven diese Idee nie spezifiziert oder auf die einschlägige Literatur zurückgegriffen hat; dabei wären die Arbeiten der politikwissenschaftlichen und bisher einzigen weiblichen Nobelpreisträgerin für Wirtschaftswissenschaften, Elinor Ostrom, geradezu prädestiniert. Sie hat sich Zeit ihres Lebens mit genau diesem Regierungstypus, der „polycentric governance“, theoretisch wie empirisch beschäftigt (Ostrom 1990, 2008, 2010).

9 Diese Position ist nicht - wie oft vorschnell unterstellt wird - identisch mit der Vorstellung des Primats der Politik. Er setzt voraus, dass zentrale Bereiche der Gesellschaft (wie etwa Ökonomie, Bildung, Wissenschaft, Religion) faktisch und fundamental der Politik unterworfen sind. In der politischen Gesellschaft dagegen besteht nur die virtuelle Möglichkeit eines solchen Primats, die durch das Gewaltmonopol und die damit gegebene Fähigkeit für verbindliches Entscheiden gegeben ist. Die Teilsysteme der Gesellschaft sind jedoch immer und unvermeidlich durch eine erhebliche Anzahl politischer Regelungen reguliert und die Intensität dieser Regulierung muss immer politisch entschieden werden. Welche Rolle politische Regulierungen bei der Ausdifferenzierung gesellschaftlicher Teilsysteme in der Moderne gespielt hat, ist u. a. von Huf (1998a, 1998b) nachgezeichnet worden. Aber wie umfassend Politik die Dynamiken der Teilsysteme reguliert und ihren Vorstellungen unterstellt, wissen wir nicht erst seit Bologna! Und die Diskussion um die Rolle, ja den Primat der Politik gegenüber den (unregulierten) Finanzmärkten verdeutlicht, dass in der globalisierten Welt politische Entscheidungen verstärkt eingefordert werden. 
verkürzte Politikbegriffe (Greven 2001a). Erstere reduzieren Politik auf ihren funktionalen Beitrag zur Stabilisierung bzw. Reproduktion von Gesellschaften und enthistorisieren die Politik, während letztere sie auf rationale oder problemlösende Entscheidungen fokussieren und so zu einer „Betriebswirtschaft der Politik" (Greven 2001a, S. 32) degradieren. Hier stehen vor allem die rationalistischen oder steuerungstheoretisch inspirierten policy-analytischen Konzepte bzw. Theorien im Mittelpunkt seiner Kritik (Greven 2008, 2009). Politik - so Michael Th. Grevens Vermutung - hat es zwar (ausnahmsweise) auch mit Problemlösungen und dem Kampf um Anteile an Macht zu tun; ja, der Machtbegriff ist zentral für ein angemessenes Verständnis von Politik. Macht aber - so seine Pointe - verweist auf einen „unreduzierbar kontingenten Charakter. Denn wer nach ,Macht ${ }^{6}$ strebt, der sucht die Möglichkeit, Ziele zu verwirklichen, und wer sie in einer bestimmten Konstellation , besitzt ${ }^{*}$ - ein eigentlich unzureichender sprachlicher Ausdruck -, dessen Möglichkeit, Ziele zu verwirklichen, hat sich erweitert" (Greven 2000, S. 36). Die unreduzierbare Pluralität der Absichten und Ziele in modernen Gesellschaften, die nie vollständig zu verwirklichenden Ziele, die am Widerstreben anderer abgeschliffen und verändert werden, die fehlenden ,guten' Gründe und das mangelnde Wissen für diese und gegen jene Entscheidung produzieren jene Kontingenz, die für Politik charakteristisch ist.

Die Demokratie ist diejenige politische Form, die der Kontingenz der Politik am angemessensten ist, weil der prinzipielle Relativismus der Politik hier zur Geltung kommen kann und zugleich durch institutionelle und andere Mechanismen der Selbstbeschränkung in Grenzen gehalten wird (Greven 1995). Allein solche selbstinduzierten „Fessel[n] und Bremse[n]“ (Offe 1989) relativieren die im Prinzip grenzenlose Politisierbarkeit in politischen Gesellschaften, die gleichwohl durch Politik umgestoßen werden können. Konsens oder diskursiv ermittelte Übereinstimmung sind die Ausnahme, aber nicht die Regel bei politischen Entscheidungen in der politischen Gesellschaft. Neben funktionalistischen und finalistischen Politikverständnissen betrachtet Michael Th. Greven auch deliberative als „Entpolitisierung“, weil deliberativ-diskursive Verfahren eine „Vermutung der Vernünftigkeit“ (Habermas 1992, S. 368) bzw. eine universalistische Richtigkeit unterstellen, die das prinzipielle Auseinanderfallen von Vernünftigkeit und (Mehrheits-)Entscheidung in modernen demokratischen Gesellschaften übersieht (Greven 2010, bes. S. 78-79). Die Vorstellung universaler Vernünftigkeit aber unterliegt der Illusion, dass der grundlegend plurale und agonale (Kampf)Charakter der Politik durch einen argumentativen bzw. vernunftbasierten Konsens ersetzt werden kann.

Politik wird von Michael Th. Greven - in Anlehnung an die ideengeschichtlichen Untersuchungen und die Weber-Interpretation des finnischen Politologen Kari Palonen $(1985,1998,2006)$ - vor allem als Chancenbeschaffung begriffen, als Möglichkeiten, die durch politisches Handeln eröffnet und durch politische Entscheidungen wieder geschlossen werden (Greven 1999, 2000). Was in einer spezifischen Gesellschaft als möglich betrachtet wird, ist abhängig von deren Politikprozessen, die man in drei Ebenen unterteilen kann (Greven 2001b). Die erste Ebene ist die kulturell-kognitive: Was in einer gegebenen Gesellschaft als politisch betrachtet wird, wird durch Traditionen, Gewohnheiten und Routinen bestimmt; 
diese legen den Horizont von Chancen fest und werden durch die Inklusivität von Gesellschaften geleitet: Wer als Person oder Gruppe an der Politik teilnehmen und was an Inhalten in der Politik thematisiert werden kann, was also im kulturellen Kontext politisiert werden kann, wird durch den Grad der historisch variablen Inklusivität festgelegt. Die zweite Ebene ist die institutionell-prozedurale, in der in einer Gesellschaft das faktische Potential der Politik ausprobiert wird: Politische Akteure und soziale Gruppen politisieren einen bestimmten Sachverhalt, um ihn zum Gegenstand einer politischen, also verbindlichen Entscheidung zu machen. Was politisiert und wie etwas politisiert wird, ist von den Politisierungskräften und dem -potential einer gegebenen Situation abhängig. Und umgekehrt reflektiert der gesamte Bestand des positiven Rechts das bereits politisierte Themenspektrum, insofern es durch verbindliche Entscheidungen zu Institutionen oder zu materialem Recht geronnen ist. Die dritte Ebene ist schließlich die evaluative, in der durch politische Imagination eine Gesellschaft das reflektiert, was auch noch zum Gegenstand der Politik werden könnte. Der Politikbegriff fokussiert dann vor allem auf Politisieren als etwas durch Politik bespiel- und entscheidbar zu Machendes; nur dadurch werden Chancen und Möglichkeiten eröffnet, durch die Gesellschaften ihre Gestaltungshorizonte thematisieren.

Politik ist dann weder zielgerichtete politische Steuerung, noch rationale Problemlösung, noch sich autopoietisch vollziehende Selbstreferenzialität, noch diskursiv ermittelte Wahrheit bzw. universale Vernunft, sondern ein politischer Kampf um die kontingenten Möglichkeiten, die sich im politischen Wettbewerb eröffnen. Und diese Möglichkeiten sind im Prinzip unbegrenzt, denn Politik kann (fast) alles, weil sie, mit dem Monopol der physischen Gewaltsamkeit in der Hinterhand, (fast) alle Regeln brechen, aber auch neue setzen kann. Dies macht die Ambivalenz der Politik in der politischen Gesellschaft aus. Aber wie dieser Kampf ausgeführt wird, welcher Strategien, Taktiken, Handlungsmuster und Routinen sich politische Akteure bedienen, welche sprachlichen Symbolisierungen sie nutzen, welche Mobilisierungs-, Empörungs- und Erregungstechniken sie einsetzen und wie sie Macht umsetzen - all das bleibt doch etwas unklar. Denn zentral für jede Analyse des Politikbegriffs ist, dass man zwischen einer rein funktionalen Bestimmung und dem Handlungsbegriff von Politik unterscheidet, wobei sich bei letzterem eine fast nicht zu übersehende Spannbreite von Spielarten des „PolitikTreibens" eröffnet.

Wie hat aber nun die politikwissenschaftliche, soziologische oder staatsrechtliche Literatur des ausgehenden 19. und des beginnenden 20. Jahrhunderts den Wandel zur politischen Gesellschaft im Politikbegriff reflektiert? Lassen sich in der zeitgenössischen Literatur Spuren eines Umschreibens des Politikbegriffes entdecken und welche Konturen nimmt er an? 


\section{Der Politikbegriff in der politischen Gesellschaft: Zwischen Kontingenzgenerator und Komplexitätsreduktor}

Ideengeschichtlich ist die Frage zentral, wer wann und unter welchen Bedingungen das Moment markiert hat, das einen Neuanfang im politischen Denken setzt. Der Begriff des Moments ist vielschichtig, aber er verdeutlicht einen Zeitpunkt, ab dem etwas Neues, bisher nicht Dagewesenes beginnt. Zugleich leitet er eine Dauer, einen Zeitabschnitt ein, innerhalb dessen das Neue des Moments das politische Denken und die wissenschaftliche Diskussion prägt, aber nicht unbedingt dominiert. ${ }^{10}$ Und schließlich steckt der Begriff ein Feld ab, indem sich nun (neue) Autoren positionieren, sich durch Verweise miteinander verbinden und ein „Gewebe politischer Diskurse“ (Llanque 2008) entstehen lassen. Der finnische Politologe und Ideengeschichtler Kari Palonen hat in verschiedenen historischen Studien den Wandel des Politikbegriffs in Europa untersucht und festgestellt, dass wir es zu Beginn des 20. Jahrhunderts mit einem Wandel vom Substanz- bzw. Disziplinbegriff zum Handlungsbegriff zu tun haben (Palonen 1985, 1989, 1998, 2003). Diesen Wandel will ich am Beispiel von Albert E. F. Schäffle, Karl Mannheim und Max Weber skizzieren und abschließend den Politikbegriff in Niklas Luhmanns frühem Werk nachzeichnen. Niklas Luhmann als letzter meiner Spurenleger versucht, das Überbordende der Politik, das ihr immanent innewohnende Destruktionspotential durch Strategien der Reduktion von Komplexität auszutreiben.

\subsection{Schäffles Politik als schöpferische Tätigkeit und Verflüssigung des Bestehenden}

Der deutsche Politiker, Nationalökonom und Soziologe Albert E. F. Schäffle hat 1897 - auf Wunsch der Leserschaft - in der Zeitschrift für die gesamte Staatswissenschaft einen Artikel „Über den wissenschaftlichen Begriff der Politik“ (Schäffle 1897) geschrieben, der für meine Spurensuche das Moment markiert, welches in der Ideengeschichte weitgehend unberücksichtigt blieb. ${ }^{11}$ Hier vollzieht Schäffle eine radikale Neubestimmung des Politikbegriffs, indem er ihn nicht nur vom Staatsbegriff löst, sondern zugleich als Handlungsbegriff konzipiert, der keinem ex ante bestimmten Staatszweck bzw. einer Staatsräson folgt, sondern durch seine prinzipielle Offenheit und Ziellosigkeit charakterisiert ist.

Zunächst führt Schäffle eine Trennung des Staatslebens in die Politik und durch Recht und Bürokratie geprägte Tätigkeiten ein. Der eine Teil ist das feststehende, festgeordnete und gleichmäßig sich vollziehende Staatsleben, und der andere der der „Flüssigkeit, des Werdens, der Veränderung, der erst im Einzelfalle fertig zu bringenden Entscheidung, des erst zu Schaffenden, oder der Erhaltung als eines fortgesetzten Neuschaffens“ (Schäffle 1897, S. 589). Man kann - so Schäffle weiter - den ersten Teil als „laufendes Staatsleben“ und die andere Seite „in all ihren

10 Diese Verwendung des Begriffs Moment bei Pocock 1975, Rosanvallon 1985 und Palonen 1998.

11 K. Palonen hat Schäffle in seiner Schrift über den Horizontwandel des Politikbegriffs in Deutschland erwähnt (Palonen 1985, S. 116-117) und auch der frühe N. Luhmann beschäftigt sich mit ihm in seiner politischen Soziologie (Luhmann 2010, bes. S. 43-45, 157). 
Erscheinungen als Politik“ bezeichnen (Schäffle 1897, S. 589). Die gegebenen Träger der Macht, also Gewalten, Beamte, Vertretungskörper, politisches Publikum, sind als feststehende und fixierte Machtgrößen laufendes Staatsleben, weil sie „gesamtheitliches Wollen unverrückbar festhalten und auf mehr oder weniger gleichmäßig wiederkehrende, allseitig fest normierte Bedürfnisfälle staatlicher Art" (Schäffle 1897, S. 589) konzentriert sind. In Max Webers Terminologie wäre das die rational bürokratische Herrschaft, die sich als feststehender Prozess in immer wieder neuen und sich gleichlaufend wiederholenden, fast automatischen Abläufen vollzieht. Dagegen steht die Politik für eine Tätigkeit, eine Aktivität und eine Form des menschlichen Handelns, die - dies soll länger zitiert werden - davon ausgeht,

„dass gesamtheitliches Wollen nicht ein für alle Male im voraus hergestellt und für immer gegeben ist, dass Größe, Art und Verteilung der gesamtheitlichen Macht auf verschiedene Träger wechselt, dass die Macht sorgfältig erst zu bilden und immer wieder neu zu gliedern ist, dass die Macht gebenden Kräfte immer wieder neu gesammelt werden müssen, dass nicht für jeden einzelnen Bedürfnisfall durchgreifend ein ins Einzelne durchreichendes positives Recht sich voraus aufstellen lässt, dass erst im gegebenen Fall die staatlich zweckmäßigste unter mehreren möglichen Anwendungen des Gesamtwillens und der Macht zu finden, rein mechanisch laufende Staatsthätigkeit überhaupt ausgeschlossen ist“ (Schäffle 1897, S. 589). ,[...] Gar vieles ist flüssig, erst im Werden und nach Zeit wie Umständen zu machen, daher - politisch zu erledigen“ (Schäffle 1897, S. 592).

Politik wird hier als Verflüssigung, Veränderung oder umkämpfte Bewahrung des Bestehenden verstanden. Die Macht- und Willenskonstellationen sind veränderbar und die Um- und Neugruppierung sind genuin politische Kämpfe, die als interaktive Auseinandersetzung mit ungewissem Ausgang konzipiert werden. Zeit und Umstände wandeln sich, die Kontexte der Politik variieren und eröffnen Chancen und Möglichkeiten. „Politisch erledigen“ heißt, eine verbindliche Entscheidung zu treffen, die dann das Bewegte festhält und zur verwaltenden Staatstätigkeit werden lässt. Zwar bewegt sich die Politik innerhalb von strengen Gesetzen (den positiven Gesetzen und dem Sittengesetz), aber sie hat

„ihren breitesten Boden und ihren reichsten Inhalt auf dem Boden der Fort-, Umund Rückbildung des Bestehenden. Dafür gilt es, gesamtheitliches Wollen zu erzeugen und die zielführenden Mittel zu gewinnen, statt feststehendes Recht mit schon gegebenen Mitteln nach zeitweilig unveränderten Regeln zur Geltung zu bringen. Der für jede Zeit überaus inhaltsreiche und weite Kreis der Entwickelung des Ganzen im Einzelnen und des Einzelnen im Ganzen aus der Gegenwart heraus in die Zukunft hinein, das Entstehenlassen aus dem Bestehenden heraus, das Wachstum und der Verfall der Volksgemeinschaften sind die an Politik reichsten Gebiete staatlicher Thatsachen" (Schäffle 1897, S. 593).

Die hauptsächliche Bedeutung der Politik liegt in der Kontingenzproduktion sowohl bei der Neuformierung von Machtverhältnissen als auch in der Policyproduktion - beides eröffnet neue Möglichkeiten und neue Spielräume. Das Schöpferische, Neue, Flüssige ist als reiner Gegensatz zur Routine und zur Wiederholung 
in bürokratischen Handlungsmustern konzipiert, und Verflüssigen wird nun zum alleinigen Merkmal der Politik. Sie ist durch keine Substanz, keine vorgegebenen Zwecke und Ziele, wie etwa die Staatsräson (Meineke 1929; Münkler 1987), gekennzeichnet, sondern durch die Qualität des Neue-Spielräume-Schaffens. Politische Entscheidungen sind dann die Mechanismen, die diese Offenheit für kurze Dauer schließen und stabile Ordnungsmuster kreieren, die aber durch Politik sofort wieder hinterfragt und in Bewegung gesetzt werden. Das Verhältnis von Öffnen und Schließen ist bei Schäffle asymmetrisch gedacht: Politik verkörpert den Primat des Öffnens, während Entscheiden und das Abschließen der Situation eine unvermeidliche, aber sekundäre Rolle spielt.

Auffällig an dem Text sind die verwendeten Begriffe, die das bisherige Repertoire umschreiben (Palonen 1998, 2001). Schäffle verwendet an vielen Stellen den Begriff „Politik treiben“, der dann später bei M. Weber so prominent und in „Politik als Beruf“ (Weber [1919] 1992) eine zentrale Rolle spielen wird. Schäffle hat dies bereits vorweggenommen. Denn Staatsoberhäupter und Minister „treiben Politik“ (Schäffle 1897, S. 583), sofern sie neue Machtkonstellationen bewirken wollen; wer nur repetitive Tätigkeiten und Routinen vollzieht, der treibt nicht schon, wenigstens nicht ausschließlich und gänzlich, Politik. Dagegen wird die von politischen Parteien betriebene Tätigkeit des Aufbaus und der Konzentration von politischer Macht für bestimmte Entscheidungen und v. a. die politische Agitation „als ein hervorragend ,politisches Treiben“ angesehen“ (Schäffle 1897, S. 583). Und an anderer Stelle hat er festgehalten, dass Beamte, die eigentlich keine Politiker sind, dennoch „Politik treiben“, wenn sie ihre Vorgesetzten auf Missstände im Volksleben aufmerksam machen (Schäffle 1897, S. 584). Hat Weber den Text von Schäffle womöglich doch gekannt, hat er diese Begrifflichkeit wegen ihrer Anschaulichkeit und wegen seines ähnlich gelagerten Denkens einfach übernommen, ohne sich der Quelle bewusst zu sein? Oder hat er sie - was mir nicht ausgeschlossen erscheint - einfach nicht angegeben? ${ }^{12}$ Wie dem auch sei: Politik ist nichts Feststehendes, also keine Substanz, kein vorgegebener Wille, kein feststehendes Gemeinwohl, weder aristotelisches „Gutes“ noch eine zweckorientierte Staatsräson, sondern ein kontingentes Phänomen, das immer erst hervorzubringen, immer erst zu Ende zu schaffen, immer erst zu entscheiden ist. Aber auch das bereits Feststehende ist der Dynamik der Verflüssigung durch Politik ausgesetzt.

Die Akteure der Politik sind nicht - wie man vermuten könnte - die obersten Regierungsorgane, sondern auch die Parteiführer, Volksvertretungen, aber auch Einzelne, die in „geselligen Kreisen“ (Schäffle 1897, S. 591) auftreten und um ihre Positionen kämpfen. Politik als Kampf - das ist nicht erst bei Weber eine zentrale Prämisse, sondern bereits bei Schäffle prominent. Zwar tauchen Interessenorganisationen oder zivilgesellschaftliche Akteure nicht explizit auf, aber „alte“ soziale Bewegungen, die Arbeiter- und Gewerkschaftsbewegungen, sind durchaus relevante Akteure, die Politik ebenso treiben wie das „politisierende Publikum“

12 K. Palonen vermutet, dass Weber den Text kannte, aber - wie auch in seinen anderen Schriften bei seinen nominalistischen Begriffsbildungen keinen Bezug auf die Ausführungen anderer AutorInnen nahm (Palonen 2001, S. 26). 
(Schäffle 1897, S. 592). Hier findet man bereits die Dreiteilung des politischen Systems in Politik, Verwaltung und Publikum, mit der dann Luhmann später in seinen systemtheoretischen Analysen des politischen Systems arbeitet (Luhmann 1968, 1981, 2000, 2010).

Zudem sieht Schäffle, dass Politik eine Tätigkeit ist, die mit Leidenschaften, Intensitäten und Emotionen operiert. Politik hat eine bestimmte Erlebnisqualität, wenn er z. B. schreibt, dass Wahlen das ganze Volk in „die politische Siedehitze des Wahlkampfes“ versetzen oder „in lichter Lohe die Rivalität“ zwischen politischen Persönlichkeiten entbrennen, seien es Parteiführer oder andere Politiker (Schäffle 1897, S. 592). Politik ist also nie ohne Leidenschaft spielbar und auch nicht denk- bzw. analysierbar.

Damit ist ein erster Markstein gesetzt: Politik und Verwaltung bzw. mechanische Staatstätigkeit lassen sich deutlich trennen und Politik ist der Teil, der sich zwar in den Institutionen und Regeln streng nach dem positiven oder dem Verfassungsrecht vollzieht, aber gleichwohl erhebliche Spielräume hat und sich auf das Verflüssigen des Alten und Gestalten des Neuen konzentriert. Hierbei eröffnet sich ein weiter Spielraum, in dem das schöpferische, kreative, zukunftsorientierte Handeln sich ausspielen lässt und sich auf die Neuformung und Beherrschung von politischen Machtkonstellationen konzentriert. Das staatliche Leben ist somit zweigeteilt: In rational-bürokratischen Vollzug einerseits und in Politik anderseits, was für damalige Verhältnisse bahnbrechend war. Und Politik vollzieht sich in den und ist begrenzt durch die zentralen Organe des Staates (Regierung, Parlament), aber vollzieht sich auch außerhalb und jenseits staatlicher Institutionen durch das „politisierende Publikum“ (Schäffle 1897, S. 592). Schäffle bezieht also auch das Publikum mit in den Begriff der Politik ein und gesteht zu, dass es eine Politik, von unten' gibt, die von außerstaatlichen Kräften ausgeht. Dabei wird Politik immer mit Leidenschaften gespielt. Sie ist nie allein rationales oder interessiertes Handeln, sondern hat immer eine emotionale, leidenschaftliche und thymotische ${ }^{13}$ Dimension, die später aus dem Politikbegriff mehr oder weniger herausdefiniert wurde. ${ }^{14}$

\subsection{Karl Mannheim und Politik als „irrationales Spiel“}

In Karl Mannheims „Ideologie und Utopie“ findet sich ein Kapitel, das mit „Kann es eine Wissenschaft von der Politik geben?“ (Mannheim [1929] 1952) überschrieben ist, sich explizit mit Schäffles Positionen auseinandersetzt und diese in eigentümlicher Weise radikalisiert. Wie Schäffle teilt er das Staatsleben in zwei Bereiche ein und differenziert sie wie folgt:

13 Diese Dimensionen betonen insbesondere Fukuyama 2006 und Sloterdijk 2006.

14 Die emotionale und leidenschaftliche Dimension der Politik ist in der Politikwissenschaft kaum systematisch thematisiert und wenn, dann außerordentlich skeptisch betrachtet und als weitgehend irrelevant abgetan worden (vgl. etwa den Sammelband von Klein u. Nullmeier [Hrsg.] 1999, in dem fast nur ich die Bedeutung von Emotionen betone und am Beispiel des Jugoslawienkrieges analysiert habe [Rüb 1999]; vgl. aber auch Brunner [Hrsg.] 2010; Walzer 1999; Holmes 1995.) 
"Jeder gesellschaftliche Prozess ist zerlegbar in festgeronnene Bestandteile, ,rationalisiertes Gebiet', und in einen ,irrationalen Spielraum', von dem die ersteren umschlossen werden. [...] Handeln beginnt erst dort, wo der noch nicht rationalisierte Spielraum anfängt, wo nicht regulierte Situationen zur Entscheidung drängen“ (Mannheim [1929] 1952, S. 99-100).

Die Bezeichnung des irrationalen Spielraumes ist bei Mannheim ein bewusst gewählter Gegenbegriff zur Weber'schen Rationalität, der sich auf alle Formen rational-bürokratischer Herrschaft bezieht. Rational sind all die Bereiche, die bereits durch Recht und Bürokratie geregelt sind und in denen Menschen nach festgelegten Regeln handeln. Der irrationale Spielraum ist dann der Bereich, in dem es keine Regeln gibt, den man gleichwohl durch Entscheidungen schließen muss. Irrational ist aber nicht identisch mit unvernünftig, erratisch, völlig beliebig, verrückt o. Ä., sondern als Gegenbegriff zu „rational“ im Sinne rationaler Herrschaft. Es muss Situationen geben, die unreguliert sind und in denen man Entscheidungen treffen kann. Das Irrationale zielt nicht nur auf die ungeregelte Situation, sondern auch auf den Mangel an vernünftigen Gründen für diese oder jene Option, die dann unvermeidlich dezisionistischen Charakter annimmt (Palonen 1985, S. 118). Denn im Bereich der Politik stehen sich „politische Wollungen “, also politische Parteien, gegenüber, die bestimmte Weltsichten haben und in der freien Konkurrenz um Anteile an politischer Macht kämpfen. „Die Machtkompetenzen im staatlichen wie im zwischen-staatlichen Leben sind im irrationalen Kampfe errungen, wo also die Entscheidungen des Schicksals ausschlaggebend sind“ (Mannheim [1929] 1952, S. 100). Und eben hier findet Politik statt bzw. wird Politik betrieben.

Mannheim entwickelt einen weiteren Gedanken, der über Schäffle hinausgeht und für die wissenschaftliche Analyse der Politik zentral ist: Er sieht klar, dass die politischen Parteien bzw. die großen ideologischen Strömungen seiner Zeit (Sozialismus, Konservatismus, Liberalismus, aber auch Faschismus und Bürokratismus) sich mit einem spezifischen Wissen wappnen. Dieses Wissen ist politisches Wissen. Die ideologischen Strömungen sind nicht nur als „Denkstile“ zu betrachten, deren Differenzen „bis in das Gebiet der Logik hineinragen“ (Mannheim [1929] 1952, S. 101) und bereits die Art der Problemstellung als auch mögliche Handlungsoptionen beeinflussen. Diese politischen Wollungen kämpfen in den irrationalen Spielräumen um Anteile an Macht, was „fließende, im Werden begriffene, sich stets umformende Strebungen und Entechelien“ ebenso zur Folge hat wie sich wandelnde Kräftekonstellationen, die in „immer unberechenbareren Kombinationen“ auftreten (Mannheim [1929] 1952, S. 101). Politisches Wissen (dazu Kettler et al. 1989) ist nicht reine Betrachtung, evidenzbasiertes oder gar wissenschaftliches Wissen, noch diskursiv ermittelte Wahrheit, sondern „Entscheidung und Sicht (hängen) unzertrennbar miteinander zusammen " (Mannheim [1929] 1952, S. 149). Politische Erkenntnis entsteht „erst im Handeln und im Sich-Auseinandersetzen mit der Welt“ und „Selbsterkenntnis und Erkenntnis des anderen (sind) unzertrennbar verflochten mit Handeln und Wollen“ (Mannheim [1929] 1952, S. 148). Erst in politischen Kampfkonstellationen entsteht politisches Wissen, das eben auch Kampfwissen und nicht nur Weltsicht ist. Hier tritt 
Handlungskontingenz ${ }^{15}$ auf, die sich aus der Unberechenbarkeit anderer Akteu$\mathrm{re}^{16}$ ebenso ergibt wie aus den parteipolitisch bzw. ideologisch festgelegten ,Denkstilen'. Manche Denkstile, wie etwa der Sozialismus, setzten auf das überraschende Moment in der geschichtlichen Entwicklung, auf die revolutionäre Situation, in der sich die Leidenschaften der Massen entladen, in der alles möglich erscheint und in der durch Politik völlig Neues geschaffen werden kann. Das „Offensein gegenüber dem günstigen Augenblick “ (Mannheim [1929] 1952, S. 115) ist hier zentral und damit das Schaffen von oder Ausnützen sich zufällig ergebender Zeitfenster. Hintergrundkontingenz ${ }^{17}$ äußert sich in der grundlegenden Umkämpftheit der durch die Denkstile bedingten unterschiedlichen Wahrnehmungen einer sozialen Situation, in die immer ein ganzer Komplex von ,Fakten', Bedeutungen, Gelegenheiten, Möglichkeiten und Handlungsoptionen eingeht und die daher nie Gegenstand einer ,objektiven' oder sachlichen Problemanalyse sein kann. Das alles ist zugespitzter Ausdruck irrationaler Politik.

Im Mittelpunkt des Mannheim'schen Denkens steht somit auch die Kontingenz des Bestehenden. Das Verflüssigen, das In-Bewegung-Setzen, das Umwerfen der gegebenen Kräftekonstellationen (mit unvorhersehbarem Ausgang), alles das ist nichts weniger als die Einführung von Kontingenz in das Gegebene. Und genau das ist es, was Politik vollzieht. Zugleich wird deutlich, dass politische Entscheidungen immer willkürlich sind in dem Sinne, dass politisches Denken und Wahrnehmen immer politisch ist, konkret: Dass Weltbetrachtung und die in sie eingehende Ideologie stets eine untrennbare Einheit von Betrachtung und potentieller Entscheidung ist, die eben nicht rational und objektivistisch ist, sondern immer ein ,irrationales' und willkürliches Element in sich trägt. Dieses Irrationale ist die unhintergehbare Entscheidungssituation, in der zwar der jeweils spezifische Denkstil einen groben Rahmen von möglichen Optionen gegenüber anderen absteckt, aber innerhalb dieses Rahmens sich Entscheidungen mehr oder weniger dezisionistisch ergeben. Denn Entscheiden heißt sich festlegen in einer Situation, die nicht durch Regeln durchstrukturiert ist, die neue Möglichkeitshorizonte eröffnet und in der es keine abschließenden Gründe gibt. Wie Schäffle, wenn auch deutlicher, sieht Mannheim Situationen, in denen „Handeln und Politik notwendig wird“ (Mannheim [1929] 1952, S. 100). Der Spielraum eröffnet neue und vielfältige Wege, die jedoch um der Ordnung Willen entschieden und zur rationalen Ordnung umgeschmolzen werden müssen. Erneut ist das Verhältnis von Schließen und Öffnen - wie bei Schäffle - asymmetrisch, aber diesmal zugunsten der Ordnungsbildung. Dies ergibt sich auch aus Mannheims weiteren Überlegungen, ob es eine Wissenschaft von der Politik geben kann. Es kann sie nicht geben, aber eine Synthese der verschiedenen politischen Denkstile durch „freischwebende“ Intellektuelle, die dann eine rationalere und planbare Politik als zentralen Ord-

$15 \mathrm{Zu}$ diesem Begriff und seinen Konnotationen vgl. Rüb 2012, bes. S. 125-128.

16 Dieser Sachverhalt wurde später in der Systemtheorie als doppelte Kontingenz ausformuliert. Vgl. dazu Parsons 1951, S. 16; Luhmann 1987, S. 148-191.

$17 \mathrm{Zu}$ diesem Begriff und seinen Konnotationen vgl. Rüb 2012, bes. S. 122-125. Er bezeichnet im Grunde das, was Palonen in Anlehnung an Machiavelli als „fortuna-Kontingenz" gekennzeichnet hat (Palonen 1998, S. 333-337 Herv. im Org.). 
nungsfaktor entwickeln. Aber immer bleibt ,irrationales ${ }^{\star}$ und grundloses Entscheiden zentrales Merkmal des politischen Handelns.

\subsection{Max Weber und Politik als Kampf um Möglichkeitshorizonte}

Kari Palonen - an ihm und seiner Interpretation des Weber'schen Werkes orientiere ich mich im Folgenden weitgehend - sieht die kleine Schrift „Politik als Beruf“ (Weber [1919] 1992) als „ein Fragment zu ,Politologischen Grundbegriffen““: (Palonen 2002, S. 9). Nach Max Webers Grundprämissen des soziologischen Denkens

„[bildet] die Soziologie [...] Typen-Begriffe und sucht generelle Regeln des Geschehens. Im Gegensatz zur Geschichte, welche die kausale Analyse und Zurechnung individueller, kulturwichtiger Handlungen, Gebilde, Persönlichkeiten erstrebt“ (Weber 1980, S. 9; Herv. im Org.).

Die Politologie kann man etwa in der Mitte zwischen beiden Positionen verorten - also als die Wissenschaft betrachten, die auch typenbildend arbeitet (hierin besteht ihr Gegensatz zur Geschichtswissenschaft), aber nicht ausschließlich auf „Typen-Begriffe“ und „generelle Regeln des Geschehens“ zielt (hierin besteht ihr Gegensatz zur Soziologie). Aus der Weber'schen Sicht müsste man dann davon ausgehen,

„dass Politik als Handeln prinzipiell jede Regel brechen und neue Regeln schaffen kann. Die Politologie wäre demgemäß eine Wissenschaft, die darauf ausgerichtet ist, die Eigenart dieser regelbrechenden und -bildenden Tätigkeit ,deutend zu verstehen““ (Palonen 2002, S. 8).

Damit tun sich neue Perspektiven auf, unter denen man Politik begreifen und analysieren kann. Während bei Albert E. F. Schäffle und Karl Mannheim vor allem begrifflich-konzeptionelle Rekonstruktionen des Politikbegriffs im Mittelpunkt standen, konzentriere ich mich bei Max Weber auf die Topoi, mit denen Weber Politik als Aktivität beschreibt, und auf die Typen von Politikern, die diese Aktivitäten je unterschiedlich betreiben. ${ }^{18}$

(i) Die Topoi bei Weber sind vielfältig, lassen sich aber auf drei wesentliche begrenzen. Zunächst sind die

(a) Verben relevant, mit denen er die Politik als Aktivität beschreibt und die von Schäffle - wie oben angedeutet - z. T. übernommen sind. Drei Verben sind zentral, nämlich Politik treiben, nach Macht streben und dicke Bretter bohren. Sie bezeichnen alle Sachverhalte, die sich in einer Denkfigur bündeln lassen: Es sind zunächst von konkreten Zwecken unabhängige Tätigkeiten, die in der Veränderung des Status Quo ihren Sinn finden, dem Öffnen von Chancen und neuen Möglichkeiten und der Veränderung der Rahmenbedingungen. Beim Bohren (Weber [1919] 1992, S. 82) ist - analog zum Streben - „die Veränderung das Primäre,

18 Meinen Überlegungen liegen v. a. die Analysen von Palonen zugrunde, der die Weber’schen Schriften, v. a. aber „Politik als Beruf“, in verschiedenen Anläufen ausgedeutet hat (Palonen 1998, 2001 und bes. 2002). 
das Ziel wird nicht genannt, vielmehr könnte als Resultat des Bohrens nur die Öffnung eines Spielraumes der Machtchancen erreicht werden, der zu unterschiedlichsten Zwecken benutzt werden könnte" (Palonen 2002, S. 128). Bohren ist eine subversive Metapher, die nur Löcher und Brüche bringt, aber keine positiven Ziele. ,Politik treiben“ (Weber [1919] 1992, S. 7, 14) meint Ähnliches: Es handelt sich um eine Aktivität, bei der das Ziel nie festgelegt sein kann, sondern die mit unsicheren Ergebnissen und Kontexten rechnen muss, aber genau diese Kontexte immer neu verändert und für neue Chancen öffnet., Politik treiben' ist - wie oben bereits erwähnt - terminologisch und in der Bedeutung ohne Hinweis auf Albert E. F. Schäffle übernommen. Und ,Streben“ nach Machtanteil „oder nach Beeinflussung der Machtverteilung“ (Weber [1919] 1992, S. 7) verweist ebenfalls auf Veränderung, aber muss immer mit Widerstand rechnen, der den Ausgang des Strebens offenhält (Palonen 1998, S. 158). Untrennbar verbunden ist damit

(b) die Metapher der Politik als Kampf, die die kleine Schrift durchzieht. Der Kampf bedeutet, „den eigenen Willen gegen einen widerstrebenden anderen, unter Orientierung an den Erwartungen des Verhaltens des anderen, durchzusetzen“" (Weber 1985, S. 463). Kampf hat es immer mit der „Kontingenz der Alterität“ (Palonen 1998, S. 161, 163) zu tun, weil die Anderen immer in ihren Aktivitäten berücksichtigt werden müssen und im Kampf Widerstand entgegensetzen. Der Kampf (mit Anderen) setzt auch die eigene Kontingenz voraus, denn man entwickelt seine eigenen Aktivitäten immer auch in der Erwartung dessen, was diese tun könnten. Die Anderen relativieren die eigene Position, die man an das unsichere, aber dennoch erwartbare Handeln der Anderen anpasst. Der Beamte, der nicht nur bei Schäffle und Mannheim, sondern auch bei Weber immer als der Gegentypus zum Politiker auftaucht, ist eben kein Politiker, weil „Kampf, Werbung um Bundesgenossen und von freiwilliger Gefolgschaft" gerade nicht zu seinem Metier gehören. Der Politiker aber ist „Kämpfer“, denn der Kampf um politische Macht ist für Weber zentral:
„Die relationale und Aktivitätsdimension des Kampfes stecken den Horizont für die Eigenständigkeit der Politik als Handeln ab, sie machen sie sogar zum Paradigma eines Handelns, in dem der Kampf anwesend ist. [...] Die spezifisch politische Form des Kampfes kann somit als ein Komplex von intentionellem Streben und Widerstre- ben und deren wechselseitigen Nebenfolgen gedeutet werden. Die Interpretation die- ser Nebenfolgen gehört aber schon zum Gegenstand des Kampfes. [...] Die besondere Nebenfolgenkontingenz des Kampfes wird durch die beiderseitige Unkontrollierbar- keit der Folgen des eigenen Handelns qualitativ noch gesteigert" (Palonen 1998, S. 167).

Der (c) Machtbegriff umfasst zwei Dimensionen: „Macht entweder als Mittel im Dienst anderer Ziele - idealer oder egoistischer - oder Macht ,um ihrer selbst willen', um das Prestigegefühl, das sie gibt, zu genießen“ (Weber [1919] 1992, S. 7). Beim Kampf um Macht geht es nicht um den Kampf der Machtlosen gegen die Machtvollen, sondern und vor allem um verschiedene Anteile an Macht bei den bereits über Macht Verfügenden (Palonen 2002, S. 49). Die bestehende Machtkonstellation zu verändern - das ist das zentrale Moment der Politik, die 
Verflüssigung von solchen Strukturen, das In-Bewegung-Bringen solcher Konstellationen und insofern eine rein formale Bestimmung. Dann geht es
„bei der Macht nur um Mögliches, nicht aber um die Verwirklichung eines Zieles, Plans oder Projekts. In diesem Sinne kann die formale Zielsetzung der Politik als Streben nach neuen Möglichkeiten für das eigene Handeln gedeutet werden. Der Formalismus Webers liegt darin, dass Politik - anstatt als ,Verwirklichung' gewisser substantieller Ziele - als Streben nach neuen Chancen für das eigene Handeln be- stimmt wird, wobei deren Inhalt im Voraus nicht festgelegt werden kann. Man könn- te von einer ,Politik-als-Chancenschaffung" sprechen“ (Palonen 2002, S. 39).

Macht ist somit eine Figur bzw. ein Topos, der sich auf einen Spielraum bezieht, konkreter: auf das Öffnen von Spielräumen und weniger auf eine faktische Konstellation. Der Kampf um Anteile an politischer Macht ist dann nicht primär Mittel für einen vorgegebenen politischen Zweck (die Realisierung eines Programms oder einer Policy), sondern vor allem und zunächst der Kampf um die (unbestimmte) Öffnung von Spielräumen. Dieser ergibt sich daraus, dass die Anderen Andere sind und im Prinzip das Gleiche wollen. Der Versuch, politisch etwas zu realisieren, also eine verbindliche Entscheidung über einen kontingenten Sachverhalt herbeizuführen, setzt den Kampf um die Möglichkeiten, um neue Chancen voraus, weil nur bei einem erfolgreichen Öffnen des Spielraums eine bestimmte Policy realisiert werden kann. Aber das Öffnen, das Machtspiel, gehorcht anderen Logiken als die Realisation eines bestimmten Programms oder einer Policy, die nur neue Chancen realisieren will (so Weber [1919] 1992, S. 51).

(ii) Die Typen der Politiktreibenden sind bei Weber ausgesprochen vielfältig und auch hier kommt seine Kontingenzorientierung zum Tragen. Der erste und wichtigste Typus ist der selbständig Leitende. Politik - so Weber gleich zu Beginn seiner Abhandlung - ist „,jede Art selbständig leitender Tätigkeit“ (Weber [1919] 1992, S. 5) oder die „planvolle Behandlung und Führung einer bestimmten Angelegenheit" (Weber 1980, S. 30). Und Politik ist entgegen der Tätigkeit von Beamten „die Leitung oder die Beeinflussung der Leitung eines politischen Verbandes, heute also: eines Staates" (Weber [1919] 1992, S. 5). Politik zielt in diesem Sinne auf die Machtanteile, die man zur Leitung des Staates erkämpfen muss. Leitung heißt, Entscheidungen darüber zu treffen, was der politische Verband bzw. Staat verwaltungs- und beamtenmäßig zu erledigen hat. Aber die Leitung selbst erfordert einen ganz anderen Geist als der der Ausführung von Entscheidungen. Leiten und Entscheiden sind eng verkoppelt, haben mit hoher Unsicherheit zu tun und sind Möglichkeiten erweiternde und ausnutzende Tätigkeiten, die zudem verantwortet werden müssen, während ausführen und verwalten unpolitisch sind.

Neben den leitenden tritt dann im Weiteren der Gelegenheitspolitiker. Das sind wir alle, „wenn wir den Wahlzettel abgeben oder eine ähnliche Willensäußerung: wie etwa Beifall oder Protest in einer, politischen' Versammlung vollziehen, eine ,politische' Rede halten usw. - und bei vielen Menschen beschränkt sich ihre ganze Beziehung zur Politik darauf“ (Weber [1919] 1992, S. 14). Erneut sind die Parallelen zu Schäffle sichtbar, der vom politischen Publikum spricht, womit beide letztlich den politischen Staatsbürgerstatus meinen, sofern er durch politische Handlungen welcher Art auch immer aktiviert wird. Gelegenheit kann gelegent- 
lich ebenso meinen wie eine Gelegenheit im Sinne einer Chance, einer Möglichkeit (Palonen 2002, S. 49). Ihm zur Seite tritt der nebenberufliche Politiker, dessen Aktivitäten intensiver sind und unter denen alle die Personen, die bestimmte Tätigkeiten im Bedarfsfalle ausüben (Vertrauensmänner, Vorstände von politischen Vereinen und Parteien, herausgehobene Mitgliedspositionen u. Ä.), aber aus der Politik nicht, ihr Leben machen' (Weber [1919] 1992, S. 14), zu verstehen sind.

Der hauptberufliche Politiker ist der Typus, dem Weber die meiste Aufmerksamkeit widmet und dem in der politischen Gesellschaft eine herausgehobene Rolle zukommt. Er unterscheidet zwei Typen: den für die Politik und den von der Politik lebenden. Ersterer macht im innerlichen Sinne sein Leben daraus, indem er den nackten Besitz der Macht genießt, oder „er speist sein inneres Gleichgewicht und Selbstgefühl aus dem Bewußtsein, durch Dienst an einer ,Sache' seinem Leben einen Sinn zu verleihen“ (Weber [1919] 1992, S. 16; Herv. im Org.). Dieser Sinn ist jedem ernsthaften politischen Menschen inne, während der von der Politik lebende aus ihr vor allem eine dauerhafte Einnahmequelle zu machen versucht. Dieses Von-der-Politik-leben ist bedeutsam, weil der Berufspolitiker durch permanente Wahlen und der damit verbundenen Unsicherheit seiner Einkommensquelle immer bedroht ist. Das Bestreben der von der Politik Lebenden geht deshalb immer dahin, diese Unsicherheit der Politik-als-Beruf zu reduzieren und sie durch andere Positionen im Staat oder in den Parteien dauerhaft abzusichern (Borchert 2003). Vom Beamten oder Verwaltungsmenschen unterscheidet sich der von der Politik Lebende eben durch die permanente Unsicherheit seiner beruflichen Position in der Demokratie. Die Folge ist Patronage: „[...] heute sind es Ämter aller Art in Parteien, Zeitungen, Genossenschaften, Krankenkassen, Gemeinden und Staaten, welche von den Parteienführern für treue Dienste vergeben werden. Alle Parteikämpfe sind nicht nur Kämpfe um sachliche Ziele, sondern vor allem auch: um Ämterpatronage“" (Weber [1919] 1992, S. 20; Herv. im Org.).

Unter historischer und vergleichender Perspektive unterscheidet Weber dann noch verschiedene Untertypen des Berufspolitikers, von denen ich nur die wichtigsten erwähnen will. Das ist zum einen der Boss, der an der Spitze einer Parteimaschinerie steht und v. a. in den USA zentral geworden ist. Durch seine USA-Reise ist dieser Typus für Max Weber prägnant geworden. Dann der Demagoge, der sowohl in der Antike, besonders aber in der neuen Zeit, in der die Bedeutung Massenmedien steigt, zentral wird. Der Demagoge ist Webers Ansicht nach „seit dem Verfassungsstaat und vollends seit der Demokratie der Typus des führenden Politikers im Okzident. Der unangenehme Beigeschmack des Wortes darf nicht vergessen lassen, dass nicht Kreon, sondern Perikles der erste war, der diesen Namen trug“ (Weber [1919] 1992, S. 33). Perikles leitete die Ekklesia des Demos von Athen und beeinflusste deren Dynamiken nach seinen Vorstellungen. Demagogie und Demokratie werden hier als unvermeidliche Zwillinge betrachtet, weil die führenden Politiker sich immer der Rede und des gedruckten Wortes bedienen müssen, um die Massen zu beeinflussen und eine gegebene Machtkonstellation ins Wanken zu bringen. Der Boss ist erneut der zugespitzte Ausdruck dieses Typus, der „keine festen Prinzipien (hat), er ist vollkommen gesinnungslos und fragt nur: Was fängt Stimmen?“ (Weber [1919] 1992, S. 54). Dieses Verhalten wird heute als „vote-seeking “ oder Stimmenmaximierung bezeichnet, wobei sich die Berufspolitiker 
oft „vollkommen gesinnungslos“ an den Stimmungen des Elektorats orientieren und keine Policies geschweige denn „Lösungen“ für gesellschaftliche Problemen realisieren. Vielmehr entwickeln sie Policies, um Stimmen zu maximieren. ${ }^{19}$

Weber hat - um zusammenzufassen - einen neuen Horizont im Politikbegriff geöffnet, indem man nun den Begriff der politics, der das politische Handeln als konflikt- und konsensorientiertes Handeln bezeichnet, in zwei neue Dimensionen bzw. Handlungstypen unterteilt (Palonen 1993): (a) Politisierung (oder politicization) verweist auf die Öffnung oder Markierung eines Sachverhaltes als politisch bespielbar zu machenden, auf den bezogen man auch anders handeln kann, der also kontingent ist. Politisierung wäre dann die Tätigkeit, die einen bisher noch nicht bespielten Gegenstand bespielbar machen will oder einen bereits bespielten, der aber im Moment nicht bespielt wird, erneut bespielbar zu machen trachtet. Und dann wäre (b) politicking derjenige Handlungstypus, der sich in dem zuvor bespielbar gemachten Raum vollzieht. Hier wäre zwar auch der Kampf um Machtanteile anzusiedeln, aber v. a. das Spiel, das um seiner selbst willen gespielt wird, als reine Performanz, als in der Gegenwart sich abspielendes „Theater“, das durch die Brillanz oder den Dilettantismus seines Spiels das politische Publikum beeindrucken und diese Beeindruckung in Anteile an politischer Macht übersetzen will. Hier kommt der Spielcharakter der Politik zum Tragen. All die Metaphern, die dies andeuten (Theater, Dramatik, Symbolik, Schauspielern etc.), sind durchaus angebracht und bezeichnen einen wichtigen Handlungstypus. Der gesinnungslose Boss ist der Prototypus dieses Spielers, der beliebig agiert und ein professioneller Opportunist sein muss; und auch der Demagoge ist ein solcher Spieler: Er manipuliert die Massen, spielt mit ihren Leidenschaften, Emotionen und politischen Regungen und macht sie für seine Spiele nutzbar. Alles ist ein Spiel mit Kontingenzen, das der Bürokratisierung trotzt und ihr entgegensteht. Aber diese Denkfiguren kennen wir seit Schäffle.

\subsection{Niklas Luhmanns Politik. Oder: Warum man mit Politik nicht zu Frauen kommt ${ }^{20}$}

Politik ist „kein Vergnügen, hat wenig damit zu tun, wie man Vorräte sammelt, sich wärmt, sich bildet oder zu Frauen kommt. Das politische Handeln hat sich im Großen und Ganzen auf den Umgang mit Worten und Symbolen bestimmter Art spezialisiert“ (Luhmann 2010, S. 265). Aber was ist Politik im Sinne von po-

19 Das ist im Übrigen die zentrale Prämisse der ökonomischen Theorie der Politik (Downs 1957), die sich gleichwohl auf diese dürre, nutzenmaximierende Aktivität zurückzieht und die Vielfältigkeiten des politischen Handelns komplett ausblendet.

20 Die folgenden Überlegungen beanspruchen nicht unbedingt Originalität; mache Aspekte sind bereits von Lange 2003, Czerwick 2001 und - wenn auch mit anders gelagerter Schwerpunktsetzung - von Wefer 2004 aufgearbeitet worden. Gleichwohl ist eine gründliche Rezeption der Luhmann'schen „Politischen Soziologie“ bisher ausgeblieben, während die „Politik der Gesellschaft" dagegen ausführlich gewürdigt wurde (vgl. etwa Hellmann u. Schmalz-Bruns [Hrsg.] 2002; Hellmann et al. [Hrsg.] 2003). Insofern rekonstruiere ich die frühen Luhmann'schen Gedankengänge, versuche aber vor allem die bisher unterbelichteten Aspekte zu verdeutlichen und verborgene Verbindungslinien zwischen Luhmann und Schäffle, Mannheim und Weber aufzudekken. 
litischem Handeln und nicht als akteurloser, sich selbst realisierender Code? Niklas Luhmann hat sich zwar an vielen Stellen - manchmal mehr und manchmal weniger sporadisch - mit der Politik und dem Politikbegriff befasst, ${ }^{21}$ aber erst 2000 erschien aus seinem Nachlass die scheinbar „endgültige“ Version der Systemtheorie der Politik, die „Politik der Gesellschaft“ (Luhmann 2000). Nun ist im Jahr 2010 erneut eine bisher unveröffentlichte Vorlesung erschienen, die Luhmann bereits 1968 gehalten hat und von A. Kieserling mit einer knappen editorischen Notiz als „Politische Soziologie“ herausgegeben wurde (Luhmann 2010). Hier zeigt sich Luhmann - im Gegensatz zur „Politik der Gesellschaft” - ganz auf der Höhe der damaligen politikwissenschaftlichen Diskussion ${ }^{22}$ und hier tauchen auch Albert E. F. Schäffle, Karl Mannheim und unvermeidlich Max Weber auf. Zwar nimmt Niklas Luhmann nur an zwei Stellen explizit auf Schäffle und Mannheim Bezug, ${ }^{23}$ aber der Einfluss von beiden - so meine Vermutung - geht erheblich weiter. Zentrale Begriffe und Konzeptionen liegen parallel zu ihnen, auch wenn sie ausgiebig durch die zeitgenössische kybernetische und systemtheoretische Literatur abgesichert werden und er sich explizit von ihnen abgrenzt; der Einfluss von Weber ist dagegen eher gering einzuschätzen. ${ }^{24} \mathrm{Um}$ welche Stellen bzw. Sachverhalte handelt es sich, an bzw. von denen Luhmann sich explizit abgrenzt? Sind es zentrale oder eher marginale Sachverhalte, die diese explizite Abgrenzung erheischen? Oder sind es theoriestrategische Ablenkungen, um die vielen Parallelen zu verwischen oder zu verdecken?

Die erste Bezugsstelle findet sich in der Diskussion über das Wesen der Politik. Hier setzt sich Luhmann von C. Schmitts „Begriff des Politischen“ ebenso ab wie von K. Mannheims Politikbegriff als Bereich des Irrationalen (Luhmann 2010, S. 36). Statt jedoch Wesensaussagen zu treffen, scheint es ihm plausibler, von einer Klärung der spezifischen Funktion der Politik auszugehen. Schäffles Betonung des „Schöpferischen“ wird abgelehnt (Luhmann 2010, S. 36, FN 43), Mannheims Verschärfung ebenfalls und dann folgt ein Zitat eines italienischen Faschisten, der Ähnliches betont. Damit ist die Sache für Luhmann aus der Welt geschafft, denn wer mit faschistischen Politikverständnissen in Verbindung gebracht wird, der ist erledigt. Was aber Luhmann eigentlich nicht ertragen kann, ist, dass Schäffle wie Mannheim keine funktionale Rückbindung der Politik vorsehen, sondern sie als sich grundlos Öffnende, sich frei bewegende und als funktionslose Handlung konzipieren.

Er setzt dem eine Position entgegen, die in der Politikwissenschaft dominant geworden ist, allerdings ohne die Luhmann'sche Pointe zu erfassen; sie wurde vielmehr systematisch negiert, verdrängt, normativ umdefiniert oder als faktische

21 Das alles aufgearbeitet und systematisiert zu haben, ist das unschätzbare Verdienst von Lange 2003; vgl. auch Czerwick 2001, die gleichwohl vor Erscheinen der „Politischen Soziologie“ schreiben mussten.

22 Das kann man für die „Politik der Gesellschaft“ nicht sagen, bei der Luhmann ab Mitte/Ende der achtziger Jahre aufgehört hat, die politikwissenschaftliche Literatur zu rezipieren.

23 Das sind die Stellen auf S. 36-37 und dann wieder auf S. 157, wobei beide nur in den Fußnoten aber immerhin - erwähnt werden.

24 Der Einfluss von Weber ist für den Machtbegriff zentral, aber nicht für den Politikbegriff, wie Czerwick (2001, S. 295) etwas vorschnell unterstellt. 
Problemlösung ausgegeben. Luhmann formuliert apodiktisch: Der Bezugspunkt einer theoretischen Bestimmung der Politik ist die „problemlösende funktionale Leistung “ (Luhmann 2010, S. 37): Aber diese Leistung vollbringt das politische System als System (und nicht durch seine faktischen Policies!), denn es wird mit den

„,offenen' Problemen der Gesellschaft konfrontiert, und es löst diese Probleme durch bindende Entscheidungen [...]. Das politische System reduziert Komplexität mithin nicht nur wie ein Organismus für sich selbst, als Bedingung seines eigenen Fortbestehens in einer überkomplexen Welt, sondern in erster Linie für seine Umwelt, die selbst den Charakter eines umfassenden Systems hat" (Luhmann 2010, S. 38; Herv. im Org.).

Bindende Entscheidung und Problem(lösung) sind formale Begriffe, denn es ist offen, um welche Probleme es sich handelt und welche thematisiert werden und welche nicht; und zugleich gilt dies auch für bindende Entscheidungen als Lösung, denn sie sind formal Entscheidungen, die so oder auch anders hätten ausfallen können. Sie sind allein als bindende Entscheidung eine Lösung, weil sich in den jeweiligen Situationen das Richtige nicht von selbst ergibt, ,ja man kann sagen: in der es keine richtige oder falsche Lösung gibt“ (Luhmann 2010, S. 41). Das „lösende" Moment ist also allein die Bindungswirkung, nicht mehr, aber auch nicht weniger. Aber diese signalisiert der Gesellschaft, dass es ein (Teil)System gibt, das bindende Entscheidungen für im Prinzip beliebige „Probleme“ mit im Prinzip beliebigen „Lösungen“ produzieren kann. Diese rein funktionale und v. a. formale Bestimmung der Funktion des politischen Systems ist dann von Teilen der Disziplin vorschnell als faktisches Problemlösen (miss)verstanden und zugleich mit Politik als politischem Handeln gleichgesetzt worden. Was Politik aber als spezifisches Handeln ist, als Aktivität von Gruppen und Individuen in bestimmten Rollen (ja, das gibt es beim frühen Luhmann noch!), das ist noch offen und erschließt sich erst in der internen Differenzierung des politischen Systems in Politik, Verwaltung und Publikum, die - wie erwähnt - bereits bei Schäffle zu finden ist.

Die Verwaltung ist der Teil des politischen Systems, der durch programmiertes Entscheiden gekennzeichnet ist. Unter Verwaltung „verstehen wir hier alle bürokratisch organisierten und programmierten Entscheidungsprozesse in Legislative, Exekutive und Justiz“ (Luhmann 2010, S. 151) und damit ist der Teil des Staatsleben bezeichnet, den Schäffle (und Mannheim) als rationalisierte Teile beschrieben haben, in denen keine Entscheidungen wegen der nicht vorhandenen Entscheidungsspielräume getroffen werden. Die Verwaltung empfängt von der Politik reduzierte Komplexität in Form vorgegebener Programme, die sie wiederum in bindende Entscheidungen für das Publikum übersetzt. Als formale Organisation ist die Verwaltung durch verschiedene Merkmale gekennzeichnet (Luhmann 2010, S. 165-172), aber ihr zentrales Merkmal ist die Abarbeitung bereits vorgegebener Prämissen. Zwar - und das unterscheidet Luhmann von Schäffle - kann sich die Verwaltung zum Teil selbst programmieren und gewinnt dadurch eine gewisse Autonomie innerhalb des politischen Systems sowohl gegenüber dem Publikum als auch der Politik. Aber davon unbenommen bleibt, dass man zwischen programmierendem und programmiertem Entscheiden systematisch trennen kann, wobei 
Letzteres v. a. der Bereich der Verwaltung ist. Programmiertes Entscheiden ist aber bei Schäffle und Mannheim kein Handeln, also auch kein Entscheiden.

Die Politik (als Handlung) dagegen hat ganz andere Aufgaben. Die funktionalen Probleme für die Politik sind doppelt gedacht. Zum einen muss die Politik mit dem Fluktuieren der politischen Unterstützung rechnen, weil die Milieus und die Quellen der Unterstützung in Bewegung geraten und durch die modernen Medien immer neue Ereignisse mit Neuigkeitswerten geschaffen werden. Die daraus potenziell resultierenden Gefahren werden geradezu beschwörend beschrieben:

\begin{abstract}
„Daraus können sehr leicht Bewegungen entstehen, die lawinenartig anschwellend das ausgewogene Gefüge funktionalspezifischer Leistungen durcheinanderbringen und das System auf primitivere Stufen der Entwicklung zurückwerfen - seien es Tendenzen zur Politisierung der Gesamtgesellschaft, also zur Aufhebung ihrer funktionalen Differenzierung, sei es ein unkontrollierbarer Ausbruch politischer Leidenschaften in Revolutionen oder standardisierten Massenbewegung. Um solchen Störungen, für die stark differenzierte Systeme typisch anfällig sind, rechtzeitig vorzubeugen, müssen die Bedingungen ständig überwacht werden, unter denen einer Verwaltung politische Unterstützung gewährt und ihr sozusagen der Gebrauch des Siegels, verbindliche Entscheidung' überlassen werden kann. Auf dieses Problem bezieht sich die Arbeit der Politik. Ihr ist an ihrer eigenen Grenze die Variabilität der politischen Unterstützung durch Publikumsrollen [...] vorgegeben” (Luhmann 2010, S. 257-258).
\end{abstract}

Und Politik - das ist dann ihre zentrale Aufgabe - hat dafür zu sorgen, dass sich diese Änderungen nicht unkontrolliert und unkoordiniert vollziehen. Die politischen Parteien als formale Organisationen spielen hier eine zentrale Rolle, weil sie trotz des Wechsels des Personals, der Programmatik, der Werte und Loyalitäten einen festen Rahmen bilden, an dem sich das Publikum orientieren kann.

Zum anderen hat es die Politik damit zu tun, der Verwaltung laufend neue und variable Vorgaben zu machen, die sie dann in rational-bürokratischen Prozessen kleinarbeitet und auf diese Weise das Publikum bindet. Die Variabilität der Entscheidungsprämissen darf aber die Verwaltung nicht überfordern und ihr gegenüber muss die Politik Komplexität reduzieren. Sie ist sozusagen der Puffer, der zwischen der extremen Variabilität der Wünsche und Vorstellungen des Publikums und der Verwaltung vermittelt, sodass diese nicht mit Komplexität überschwemmt wird. Die Positivierung des Rechts und die Variabilität des Rechtsbestandes ist bei der Auflösung vorgegebener und invariabler Staatszwecke zwar zentral, aber diese muss sich in einem von der Verwaltung noch bearbeitbaren $\mathrm{Maß}$ bewegen. Dies zu gewährleisten ist die andere Seite der Politik. Das Publikum muss also beruhigt und die Verwaltung programmiert werden - dies sind die beiden funktionalen Bezugspunkte der Politik.

Politische Prozesse oder politisches Handeln bauen Legitimität auf, indem sie die übermäßige Komplexität und Kontingenz reduzieren, die im politischen System durch das politische Publikum entstehen und sich in einer sehr spezifischen Reduktionsweise ausdrücken:

„Im Artikulieren und Verallgemeinern von Interessen und Werten, in der Beschaffung von Konsens und in der Konsolidierung konsenssichernder Vertretungsmacht, in der semantischen Arbeit an politischen ,Themen', in der Mobilisierung von Unterstüt- 
zung für Kandidaten oder Sachvorschläge, beim Lernen oder Erproben politischer Geschicklichkeiten, in der Pflege des Ansehens von Organisationen und Positionen und durch vielerlei andere ähnliche Strategien wird in einem Zuge legitime Macht aufgebaut und Komplexität reduziert“ (Luhmann 2010, S. 261).

Diese Handlungen haben einen von anderen Rollen deutlich unterschiedenen „Stil“, wie etwa der wissenschaftlichen Forschung oder der künstlerischen Gestaltung. Und dieser Stil wird v. a. durch Parteipolitiken geprägt und unterscheidet sich je nach Parteien- oder Regierungssystem. Was für spezifische Handlungen und Rollen hat Luhmann sonst noch im Auge?

Zunächst operiert Politik nicht nur als Bote, als Übermittler des Volkswillens (wie in der tradierten Demokratietheorie), der diesen Willen in die Verwaltung programmierende Entscheidungen übersetzt. Diese Kommunikationsrichtung wird durch eine gegenläufige ergänzt; die Darstellungen der Politik selbst suchen „politisches Vertrauen und Unterstützung durch das Publikum zu gewinnen und sehr oft den Willen [des Volkes, F. W. R.], den sie voraussetzt, eben dadurch erst zu schaffen " (Luhmann 2010, S. 269). Und hierbei bedient sich die Politik einer eigentümlichen Sprache und spezifischen Symbolik, die dies ermöglicht. Luhmann erwähnt hier nur einen Aspekt, die „Darstellung von etwas als etwas“ (Luhmann 2010, S. 269; Herv. im Org.): von Rüstungsausgaben als Friedenssicherung, von Patronage als Maßnahme zur Sicherung von Kompetenz in der Verwaltung, von Subventionen als Strukturverbesserung etc. Aber Forschungen dazu sind rar und erst in letzter Zeit erfolgt. ${ }^{25}$

Generell unterscheidet Luhmann dann instrumentelle und expressive Handlungsaspekte, wobei gegenüber der Verwaltung der instrumentelle und gegenüber dem Publikum der expressive Aspekt dominiert. Bei Letzterem kommt es darauf an, in „Symbolen verfestigte emotionale Einstellungen, Handlungs- oder auch Wartebereitschaften anzusprechen und zu verstärken" (Luhmann 2010, S. 270). Dies geschieht auch über (sozusagen dem Umweg der) Verwaltungsentscheidungen, indem man z. B. neue Wohltaten vor Wahlen verteilen lässt. Politik kann so je nach Bedarf - beide Aspekte einsetzen. Politische Unterstützung kann nach Luhmann instrumentell durch expressive Politik hergestellt werden, indem man entsprechende Symbolpolitiken einsetzt und politisch mit dem Publikum kommuniziert. Es entsteht eine Innenansicht, nach der die Symbole hergestellt werden, und eine Außensicht, in der sie dargestellt werden. Diese Diskrepanz ermöglicht einen „manipulativen Symbolismus“ (Luhmann 2010, S. 281), mit dem Politik operiert und die sie dem Publikum zur Schau stellt. Unterschiedliche Parteiensysteme ermöglichen unterschiedliche Umgangsformen mit diesen Problemen, wobei Mehrparteiensysteme hier eine höhere Komplexität aufbauen können als Einparteiensysteme. Sie verzichten auf übergreifende Werte und öffnen den politischen Prozess, indem nun verschiedene Parteien um den Zugang zur Macht kämpfen. Wahlen legen ziemlich exakt fest, wer wie viele Anteile an Macht bekommt und ob ein Machtwechsel stattfinden muss. Indem aber die Macht nicht Mittel für (pro-

25 Die ersten Arbeiten stammen von M. Edelmann aus dem Jahr 1964 (Edelman 1964) und sind später von ihm fortgesetzt worden (Edelman 1988). 
grammatische) Politik ist, sondern umgekehrt politische Programme zum Mittel für den Zweck des Machterwerbs werden, ${ }^{26}$ kann das politische System systemspezifische Rationalisierungen vorantreiben. Aber dieser Kalkül darf nicht offen thematisiert werden. Denn „der Verdacht, daß seine Darstellungen hergestellt sind und diese Herstellung in einer Weise motiviert ist, die nicht dargestellt wird, ist weit verbreitet und trägt zum schlechten Ruf der Politik, ja zu einem oft weit überzogenen Mißtrauen gegen alles Politische bei“ (Luhmann 2010, S. 292). Und politische Kommunikation hätte dann die Aufgabe, dieses Misstrauen kleinzuhalten und durch Symbolisierungen zu variieren. Hier wird nun der „Wille des Volkes“ zur kontingenten Größe, der sich nicht nur durch undurchschaubare Prozesse vollzieht, sondern durch (Partei)Politik selbst beeinflusst und als zu gestaltendem Material der Politik überantwortet wird. Politisches Handeln nimmt also auch diese Form der Kontingenzsteigerung und zugleich der Komplexitätsreduktion an.

Umgekehrt hat Politik die Aufgabe, ein „Mindestmaß an politischer Schwerhörigkeit" (Luhmann 2010, S. 294) zu realisieren, weil das Wünschen und Fordern des Publikums die Kapazität des politischen Systems überfordert. Auch bei der Wahl von politischen Optionen weiß die Politik nicht, wie der hochkomplexe Wählermarkt reagiert und welche Optionen das Publikum vorzieht. Hier orientiert sich die Politik an „Symptomen“ (Luhmann 2010, S. 294) und beginnt mit Symptomen statt mit Fakten zu kalkulieren. Und genau dieses Spiel mit Symptomen und Symbolen, also deren Konstruktion, deren Darstellung mit Hilfe der Medien und deren Wirkungskontrolle, die erneut über Symptome und Symbole erfolgt, gehören untrennbar zum Instrumentarium des politischen Handelns. Obwohl Luhmann hochinteressante Aspekte der Politik bzw. des politischen Handelns formuliert, hängt er ihr seine funktionalistische Zwangsjacke um, die sie auf systemspezifische Funktionen reduziert.

Hier deutet sich bereits die Wende zu einem postmodernen Politikbegriff an, der aber erst in seinem Spätwerk „Die Politik der Gesellschaft“ radikal vollzogen wird. In der „Politischen Soziologie“ hat die Politik eine noch weitgehend selbstständige und autonome Bedeutung, die doppelt funktional rückgebunden ist: Sie soll zum Ersten die hoch komplexen und unkontrollierbaren Bewegungen des politischen Publikums kontrollieren und zugleich die Überforderung des Systems durch die Ansprüche des Publikums verhindern. Und zum Zweiten muss sie die Verwaltung mit Programmen programmieren, in denen Komplexität bereits so weitgehend reduziert ist, dass sie in bürokratisch zu bewältigende Verwaltungsaufgaben geformt werden. Hierbei kann die Politik eigene Selektivitäten ausüben und beide funktionalen Bezugspunkte bearbeiten, wobei ihr hierbei ein weiter Spielraum von unterschiedlichen Aktivitäten und Praktiken eingeräumt wird. Erst in der „Politik der Gesellschaft“ wird die post-moderne Variante deutlich formuliert: Der eigenständige Spielraum reduziert sich und Politik stellt um von „zielorientierter Rationalität“ auf „zeitorientierte Reaktivität“ (Luhmann 2000, S. 142). Zielgerichtete Gestaltungs- oder gar Steuerungsansprüche sind radikal aufgegeben und Politik zieht sich in ihr Schneckenhaus zurück und wird nur noch

26 Die Referenz ist hier natürlich Downs ökonomische Theorie der Politik (Downs 1957) und alle, die ihm gefolgt sind. 
situativ und reaktiv tätig. Aber wenn sie tätig wird, dann als Spiel, bei dem v. a. mit Worten und Symbolen reagiert wird und bei dem es - auch im Rahmen der funktionalistischen Beschränkungen - nicht mehr um die Realisation normativer oder programmatischer Prämissen durch die Programmierung der Verwaltung geht, sondern allein um vorwiegend symbolische Reaktionen auf systemische Eigendynamiken der Teilsysteme einer (globalisierten) Gesellschaft.

\section{Schlussbemerkung}

Aus der ideengeschichtlichen Spurensuche wurde das Umschreiben des Politikbegriffs im Übergang von der vorpolitischen zur politischen Gesellschaft deutlich. Politik als Handeln konzentriert sich auf den Bereich, in dem es um die schöpferische Tätigkeit des Verflüssigen und Neuschaffens von gesellschaftlichen Wirklichkeiten geht und wo Entscheidungen in einem ungeregelten Spielraum getroffen werden müssen. Das Verhältnis von Öffnen und Schließen des Kontingenzraumes wird zwar unterschiedlich akzentuiert, aber politisches Handeln ist beides zugleich und kann im Prinzip jeden Sachverhalt erfassen. Fundamentalpolitisierung durch „politicization“ ist zentral für politisches Handeln, wobei sich demokratische und diktatorische politische Gesellschaften hinsichtlich der Politisierungstiefe und der -formen drastisch unterscheiden. Aber immer fehlt eine rationale Grundlage, weil es für diese und gegen eine andere Entscheidung letztlich keine entscheidenden Gründe gibt. Ihr ist ein dezisionistisches oder - wie Mannheim sagt - irrationales Moment immanent. Und Chancen und Möglichkeiten ergeben sich nur im politischen Kampf, weil allein hier neue Macht- und Herrschaftskonstellationen entstehen, die die alten verändern und neue konstituieren und aus denen sich erneut kontingente Möglichkeiten ergeben. Politik hat eine Erlebnisqualität, die von den politiktreibenden Personen und Gruppen als positiv betrachtet wird.

Diese Positionen sind weitgehend mit denen von Greven identisch, wie er sie in der „Politischen Gesellschaft" und in anderen Schriften entwickelt hat, auch wenn bei ihm die Erlebnisqualität nur eine sehr untergeordnete Rolle spielt. Aber das, was Greven eher deduktiv und unter Bezugnahme auf Weber bzw. Palonens Weberinterpretation als für Politik konstitutiv hält, ist mit den ideengeschichtlichen Umschreibungen doch erstaunlich kompatibel. Was er übersieht, ist die Form des politischen Handelns, die Palonen als politicking bezeichnet und die in der reinen Performanz, im zweckfreien Spiel zum Ausdruck kommt. Aber immer geht dieser Politikbegriff noch von der (impliziten oder expliziten) Vorstellung aus, dass Politik gestaltend in die Gesellschaft eingreift und sie nach bestimmten Vorstellungen formt. Die politische Gesellschaft ist politische Gesellschaft insofern, als Politik die Gesellschaft mit einem Dauergerüst von verbindlichen Entscheidungen durchzieht, die alle funktionalen Teilbereiche formt und ihre Dynamiken beeinflusst. Durch das Gewaltmonopol kann Politik im Prinzip auch die funktionale Differenzierung von Gesellschaften aufheben und die sich daraus ergebenden (Eigen)Dynamiken der jeweiligen Teilsysteme außer Kraft setzen. Diese Vorstellung liegt auch der „Politischen Soziologie“ von Luhmann zugrunde, die mit den anderen erwähnten Autoren als Ausdruck eines Politikbegriffs der Moderne betrachtet werden kann. 
Ein post-moderner Politikbegriff zeichnet sich erst in Luhmanns „Politik der Gesellschaft" ab. Denn hier wird das gestaltende, aktive Moment der Politik negiert und apodiktisch formuliert, dass Politik von zielorientierter Rationalität auf zeitorientierte Reaktivität umstellt (Luhmann 2000, S. 142). Die Politiktreibenden werden dann zu von den Verhältnissen Getriebenen, die in immer neuen Schüben auf nicht mehr grundlegend zu beeinflussende Dynamiken der Umwelt der Politik reagieren. Politik stellt dann vom Verflüssigen und Neukonstituieren der gesellschaftlichen Strukturen um auf den Tanz auf dem Vulkan der globalisierten Welt. Sie politisiert weiter, sie treibt Politik, sie kämpft um Machtanteile im politischen System und erfreut sich an der Erlebnisqualität des Politischen. Aber sie kann nur noch um Macht in den Verhältnissen kämpfen, während sie die Macht über die Verhältnisse verloren hat. ${ }^{27}$ Die im Prinzip allmächtige Politik wäre dann ohnmächtig geworden.

\section{Literaturverzeichnis}

Beyme, Klaus von. 1991. Theorie der Politik im 20. Jahrhundert. Von der Moderne zur Postmoderne. Frankfurt a. M.: Suhrkamp Verlag.

Bolsinger, Eckard. 1998. Was ist Dezisionismus? Rekonstruktion eines autonomen Typus politischer Theorie. Politische Vierteljahresschrift 39: 471-502.

Borchert, Jens. 2003. Die Professionalisierung der Politik. Zur Notwendigkeit eines Ärgernisses. Frankfurt a. M.: Campus Verlag.

Bracher, Karl-Dietrich. 1964. Deutschland zwischen Demokratie und Diktatur. Beiträge zur neueren Politik und Geschichte. Bern/München/Wien: Scherz Verlag.

Brunner, José (Hrsg.). 2010. Politische Leidenschaften. Zur Verknüpfung von Macht, Emotion und Vernunft in Deutschland. Tel Aviver Jahrbuch für deutsche Geschichte 38. Göttingen: Wallstein Verlag.

Czerwick, Edwin. 2001. Politik als System: Zum Politikverständnis der Systemtheorie. In Moderne Politik. Politikverständnisse im 20. Jahrhundert, Hrsg. Hans J. Lietzmann, 287-310. Opladen: Leske \& Budrich.

Downs, Anthony. 1957. An Economic Theory of Democracy. New York: Harper \& Row. Edelman, Murray. 1964. The Symbolic Uses of Politics. Urbana: University of Illinois Press. Edelman, Murray. 1988. Constructing the Political Spectacle. Chicago: University of Chicago Press.

Fukuyama, Francis. 2006. The End of History and the Last Man. New York: Free Press.

Greven, Michael Th. 1974a. Systemtheorie und Gesellschaftsanalyse. Kritik der Werte und Erkenntnismöglichkeiten in Gesellschaftsmodellen der kybernetischen Systemtheorie. Darmstadt/Neuwied: Luchterhand.

Greven, Michael Th. 1974b. Zur Konstitutionsproblematik politischer Theorie am Beispiel der kybernetischen Systemtheorie. Kölner Zeitschrift für Soziologie und Sozialpsychologie 1: 50-74.

27 Diese und die folgende Formulierung sind Paraphrasierungen, die auf Christian Meiers Analysen über den Zerfall der Macht des Diktators Caesar zurückgehen (Meier 1986, bes. S. 24-25; vgl. auch Meier 1980, S. 23-26). 
Greven, Michael Th. 1990. Die politische Gesellschaft als Gegenstand der Politikwissenschaft. Ethik und Sozialwissenschaften. Streitforum für Erwägungskultur 2: 223-228.

Greven, Michael Th. 1992. Über demokratischen Dezisionismus. In Sprache und Politische Kultur in der Demokratie. Hans Gerd Schumann zum Gedenken, Hrsg. Dieter Emig, Christoph Hüttig und Lutz Raphael, 193-206. Frankfurt a. M./Berlin/Bern/New York/ Paris/Wien: Peter Lang Verlagsgruppe.

Greven, Michael Th. 1995. Fesseln und Bremsen - bei Montesquieu. In Politik - Verfassung - Gesellschaft. Traditionslinien und Perspektiven, Hrsg. Peter Nahamowitz und Stefan Breuer, 69-81. Baden-Baden: Nomos Verlag.

Greven, Michael Th. 1999. Die politische Gesellschaft. Kontingenz und Dezision als Probleme des Regierens und der Demokratie. Opladen: Leske + Budrich.

Greven, Michael Th. 2000. Kontingenz und Dezision. Beiträge zur Analyse der politischen Gesellschaft. Opladen: Leske + Budrich.

Greven, Michael Th. 2001a. Anmerkungen zur Kritik eines funktionalistischen und finalistischen Politikbegriffes. In Moderne Politik. Politikverständnisse im 20. Jahrhundert, Hrsg. Hans J. Lietzmann, 331-340. Opladen: Leske + Budrich.

Greven Michael, Th. 2001b. Dimensions of Politics. A Critique of the Common Onedimensional Concepts of Politics. In Finnish Yearbook of Political Thought 5, Hrsg. Ihalainen, Pasi, 89-110. Jyväskylä: University of Jyväskylä Press.

Greven, Michael Th. 2008. „Politik“ als Problemlösung und als vernachlässigte Problemursache. Anmerkungen zum Verhältnis zwischen Policy-Forschung und einem verändertem Reformverständnis in Gesellschaft und Politikwissenschaft. In Staat und Gesellschaft - fähig zur Reform? 23. wissenschaftlicher Kongress der Deutschen Vereinigung für Politische Wissenschaft, Hrsg. Klaus-Dieter Wolf, 329-339. Baden-Baden: Nomos Verlag.

Greven, Michael Th. 2009. Deutsche Policy-Forschung aus kritischer Perspektive. In Lehrbuch der Politikfeldanalyse 2.0, Hrsg. Klaus Schubert und Nils C. Bandelow, 457-473. München: Oldenbourg Verlag.

Greven, Michael Th. 2010. Verschwindet das Politische in der politischen Gesellschaft? Über Strategien der Kontingenzverleugnung. In Das Politische und die Politik, Hrsg. Thomas Bedorf und Kurt Röttgers, 68-88. Frankfurt a. M.: Suhrkamp Verlag.

Habermas, Jürgen. 1992. Faktizität und Geltung. Frankfurt a. M.: Suhrkamp Verlag.

Hellmann, Kai-Uwe, und Rainer Schmalz-Bruns (Hrsg.). 2002. Theorie der Politik. Niklas Luhmanns politische Soziologie, Frankfurt a. M.: Suhrkamp Verlag.

Hellmann, Kai-Uwe, Karsten Fischer, und Harald Bluhm (Hrsg.). 2003. Das System der Politik: Niklas Luhmanns politische Theorie. Opladen: Westdeutscher Verlag.

Hirschman, Albert O. 1987. Leidenschaften und Interessen: politische Begründungen des Kapitalismus vor seinem Sieg. Frankfurt a. M.: Suhrkamp Verlag.

Holmes, Stephen. 1995. Passions and Constraint. On the Theory of Liberal Democracy. Chicago/London: University of Chicago Press.

Huf, Stefan.1998a. Sozialstaat und Moderne. Modernisierungseffekte staatlicher Sozialpolitik. Berlin: Duncker \& Humblot.

Huf, Stefan. 1998b. Sozialstaat und Marktökonomie - oder: Wie voraussetzungsvoll ist funktionale Differenzierung? Soziale Systeme 4: 359-387.

Kettler, David, Volker Meja, und Nico Stehr. 1989. Politisches Wissen. Studien zu Karl Mannheim. Frankfurt a. M.: Suhrkamp Verlag. 
Klein, Ansgar, und Frank Nullmeier (Hrsg.). 1999. Masse - Macht-Emotionen. Zu einer politischen Soziologie der Emotionen. Wiesbaden: Westdeutscher Verlag.

Lange, Stefan. 2003. Niklas Luhmanns Theorie der Politik. Eine Abklärung der Staatsgesellschaft. Opladen: Westdeutscher Verlag.

Llanque, Markus. 2008. Politische Ideengeschichte. Ein Gewebe politischer Diskurse. München/Wien: Oldenbourg Verlag.

Luhmann, Niklas. 1968. Zur Soziologie des politischen Systems. In ders.: Soziologische Aufklärung 1: Aufsätze zur Theorie sozialer Systeme, 232-252. Opladen: Westdeutscher Verlag.

Luhmann, Niklas. 1981. Politische Theorie im Wohlfahrtsstaat. München/Wien: Olzog Verlag.

Luhmann, Niklas. 1987. Soziale Systeme. Grundriß einer allgemeinen Theorie. Frankfurt a. M.: Suhrkamp Verlag.

Luhmann, Niklas. 2000. Die Politik der Gesellschaft. Frankfurt a. M.: Suhrkamp Verlag.

Luhmann, Niklas. 2010. Politische Soziologie. Frankfurt a. M.: Suhrkamp Verlag.

Mannheim, Karl. [1929] 1952. Ideologie und Utopie. Frankfurt a. M.: Verlag G. SchulteBulmke.

Mayntz, Renate, und Fritz W. Scharpf (Hrsg). 1995. Gesellschaftliche Selbstregulierung und politische Steuerung. Frankfurt a. M./New York: Campus Verlag.

Meier, Christian. 1980. Die Ohnmacht des allmächtigen Diktators Caesar. Drei biographische Skizzen. Frankfurt a. M.: Suhrkamp Verlag.

Meier, Christian. 1986. Caesar. München: Deutscher Taschenbuch Verlag.

Meineke, Friedrich. 1929. Die Idee der Staatsräson in der neueren Geschichte. München/ Berlin: Oldenbourg Verlag.

Merkel, Wolfgang. 2010. Systemtransformation. Eine Einführung in die Theorie und Empirie der Transformationsforschung. Wiesbaden: VS Verlag für Sozialwissenschaften.

Merton, Robert K. 1980. Auf den Schultern von Riesen. Ein Leitfaden durch das Labyrinth der Gelehrsamkeit. Frankfurt a. M.: Syndikat Verlag.

Münkler, Herfried. 1987. Im Namen des Staates. Die Begründung der Staatsraison in der frühen Neuzeit. Frankfurt a. M.: S. Fischer Verlag.

Offe, Claus. 1989. Fessel und Bremse. Moralische und institutionelle Aspekte, intelligenter Selbstbeschränkung'. In Zwischenbetrachtungen. Im Prozeß der Aufklärung, Hrsg. Axel Honneth, Thomas McCarthy, Claus Offe und Albrecht Wellmer, 739-774. Frankfurt a. M.: Suhrkamp Verlag.

Ostrom, Elinor. 1990. Governing the Commons. The Evolution of Institutions for Collective Action. New York/Cambridge: Cambridge University Press.

Ostrom, Elinor. 2008. Polycentric Systems as one Approach for Solving Collective-Action Problems. In Climate Change and Sustainable Development. New Challenges for Poverty Reduction, Hrsg. Mohamed A. Salih, 17-35. Cheltenham: Edward Elgar Publishing.

Ostrom, Elinor. 2010. Beyond Markets and States: Polycentric Governance of Complex Economic Systems. American Economic Review 100: 641-672. doi: 10.1257/aer.100.3.641.

Palonen, Kari. 1985. Politik als Handlungsbegriff. Horizontwandel des Politikbegriffs in Deutschland 1890 bis 1933. Helsinki: Finnish Society of Sciences and Letters.

Palonen, Kari. 1989. Die Thematisierung der Politik als Phänomen. Eine Interpretation der Geschichte des Begriffs Politik im Frankreich des 20. Jahrhunderts. Helsinki: Societas Scientiarum Fennica. 
Palonen, Kari. 1993. Introduction: From Policy and Polity to Politicking and Politicization. In Reading the Political. Exploring the Margins of Politics, Hrsg. Kari Palonen und Tuija Parvikko, 6-16. Helsinki: Finnisch Political Science Association.

Palonen, Kari. 1998. Das ,Webersche Moment'. Zur Kontingenz des Politischen. Opladen: Westdeutscher Verlag.

Palonen, Kari. 2001. Politik statt Ordnung: Figuren der Kontingenz bei Max Weber. In Moderne Politik. Politikverständnisse im 20. Jahrhundert, Hrsg. Hans J. Lietzmann, 9-21. Opladen: Leske + Budrich.

Palonen, Kari. 2002. Eine Lobrede für Politiker. Ein Kommentar zu Max Webers „Politik als Beruf“. Opladen: Leske + Budrich.

Palonen, Kari. 2003. Four Times of Politics: Policy, Polity, Politicking, and Politization. Alternatives: Global, Local, Political 28: 171-186.

Palonen, Kari. 2006. The Struggle with Time. A Conceptual History of 'Politics' as Activity. Münster/Hamburg/London: LIT Verlag.

Parsons, Talcott. 1951. General Statement. In Toward a General Theory of Action, Hrsg. Talcott Parsons und Edward Shils, 3-29. Cambridge: Cambridge University Press.

Pocock, John G. A. 1975. The Machiavellian Moment. Princeton: Princeton University Press. Rosanvallon, Pierre. 1985. Le Moment Guizot. Paris: Éditions Gallimard.

Rüb, Friedbert W. 1999. Rückkehr zur Barbarei. Nationalismus, ethnische Konflikte und Genozid im ehemaligen Jugoslawien. In Masse - Macht - Emotionen. Zu einer politischen Soziologie der Emotionen, Hrsg. Ansgar Klein und Frank Nullmeier, 40-65. Wiesbaden: Westdeutscher Verlag.

Rüb, Friedbert W. 2012. Politische Entscheidungsprozesse, Kontingenz und demokratischer Dezisionismus. Eine policy-analytische Perspektive. In Politik und Kontingenz, Hrsg. Katrin Toens und Ulrich Willems, 117-142. Wiesbaden: Springer VS.

Schäffle, Albert E. F. 1897. Über den wissenschaftlichen Begriff der Politik. Zeitschrift für die gesamte Staatswissenschaft 4: 579-600.

Sloterdijk, Peter. 2006. Zorn und Zeit. Frankfurt a. M.: Suhrkamp Verlag.

Walzer, Michael. 1999. Vernunft, Politik und Leidenschaft. Defizite liberaler Theorie. Frankfurt a. M.: S. Fischer Verlag.

Weber, Max. [1919] 1992. Politik als Beruf. Stuttgart: Reclam Verlag.

Weber, Max. 1980. Wirtschaft und Gesellschaft. Grundriß der Verstehenden Soziologie. Tübingen: Mohr Siebeck Verlag.

Weber, Max. 1985. Über einige Kategorien der verstehenden Soziologie. In Gesammelte Aufsätze zur Wissenschaftslehre, Hrsg. Johannes Winckelmann, 427-474. Tübingen: Mohr Siebeck Verlag.

Wefer, Matthias. 2004. Kontingenz und Dissens. Postheroische Perspektiven des politischen Systems. Wiesbaden: VS-Verlag für Sozialwissenschaften.

\section{Autorenangaben}

Prof. Dr. Friedbert W. Rüb,

Humboldt-Universität zu Berlin, Institut für Sozialwissenschaften, Lehrbereich Politische Soziologie und Sozialpolitik, Unter den Linden 6,

10099 Berlin,

friedbert.rueb@sowi.hu-berlin.de 